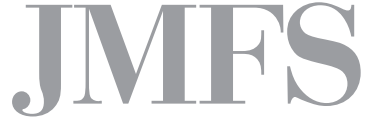

Journal of Management and Financial Sciences
Volume XIII

Issue 35 (December 2018)

pp. $93-116$

Warsaw School of Economics

Collegium of Management and Finance

Marzenna Dębowska-Mróz,

Ewa Ferensztajn-Galardos,

Renata Krajewska,

Andrzej Rogowski

Faculty of Transport and Electrical Engineering

University of Technology and Humanities in Radom

\title{
Analysis of the use of public transport in Radom
}

\begin{abstract}
An efficient transport system in cities requires rational separation of transport tasks. Determining the mode of travel and preferences of the choice of means of transport enables shaping commuting behaviours. Research on the mobility of public transport is of particular importance. Filling measurements are the basis of many analyzes, enabling the characteristics of passenger flows in the quantitative and qualitative aspect, performance of transport efficiency assessment of individual lines. The aim of the article is to present the results of a survey conducted among drivers of public transport buses. These tests included information on filling and passenger exchange in the means of transport of individual bus lines in Radom. The results of the surveys carried out in households were also presented, thanks to which the motivation behind source-destination journeys of the inhabitants of Radom with the use of public transport was analyzed.
\end{abstract}

Keywords: bus, commute, public transportation, vehicle occupancy indicator, bus occupancy, traffic, transportation demand, transportation modes, travel behaviour, travel demand, average number of journeys, destination of journeys

JEL Classification Codes: R41, R410 


\section{Introduction}

Transport in urban agglomerations plays an important role and leads to an increase or decrease in the attractiveness and development of a given region. The spread of cities, which is common in most agglomerations, causes changes in the requirements for satisfying transportation needs of passengers [Morfoulaki, M., Tyrinopoulos, Y., Aifadopoulou, G., 2007; Friman, M., Fellesson, M., 2009; Eboli, L., Mazzulla, G., 2009; Guirao, B., García-Pastor, A., López-Lambas, M. E, 2016; Wen-Tai Laia, Ching-Fu Chen, 2011]. This causes an increase in the need for fast, safe and efficient mobility. Meeting this trend is extremely difficult and requires proper management and organization of urban transport [Banister D., 2008].

The experience of large urban agglomerations shows that it is important to create integrated public transport systems, both in the country and in the world (from the point of view of adapting public transport to the requirements of passengers) [Givoni M., Banister D., 2010; Hine J., 2000; Hull A., 2005; Ibrahim M.F., 2003; Preston J., 2010]. Integrated actions, both in the spatial and functional aspects of the public transport system, will contribute to increasing the attractiveness of urban public transport and the abandonment of individual transport

Public transport, as part of the transport sector, is subject to exactly all the laws of transport economics, despite strong public interference. Globally, the analysis of demand for public transport services shows that they are determined by the following factors:

- the number of city residents;

- the level and structure of employment;

- the number of pupils in secondary schools;

- the demographic structure of the population: the share of the population aged under 18, aged 18-65 and over 65;

- the size of the area covered by the public transport network;

- the spatial structure of the city;

- the place of the city in the urban agglomeration and the degree of its connection with the suburban area;

- the level of affluence of inhabitants;

- the level of individual motorization and the degree of car use;

- the quality of public transport services;

- the level of charges for public transport services.

and also [Rudnicki, 1991]:

- the spatial and functional structure of the city, including the functions performed by the city, its level of development, the distribution of various institutions, economic, commercial, service, scientific and cultural entities related to tourism and recreation, health protection and their mutual placement, and also the spatial divergence between places of residence and places of work; 
- the level of activity of the population;

- the scope and size of free time of the population.

Transport difficulties in contemporary cities are primarily the result of increased traffic and congestion on roads. Despite prioritizing means of public transport, in practice the effects of congestion are most felt in public transport. The desire to increase the attractiveness of public transport and keep as many passengers as possible leads to the use of various methods and means of privileging it in urban traffic.

Determination of the method of journey implementation and preferences of the choice of means of transport and quality expectations reported by persons performing journeys is one of the stages of the process of shaping transport behaviour in relation to the selected spatial scope of the city [Starowicz, 2007].

Therefore, it is very important to study the motion of public transport and its main goals are [Tracz, 1984]:

- assessment of traffic conditions of public transport means - including measurements similar to measurements for means of individual transport;

- assessment of the passenger service quality - including measurements of: driving times, punctuality, regularity, frequency and occupancy;

- assessment of the effectiveness of changes in traffic organization for the privilege of collective transport;

- obtaining data for the design of lines and stops - including measurements of passenger flows and time of passenger exchange at stops.

The paper presents the results of research on the use of public transport in Radom, source-destination motivation of journeys with the use of public transport, taking into account the age and sex of travelers. The authors presented the original method of measuring the bus occupancy vehicles on the analysis of the opinion of bus drivers.

\section{Measurements of public transport vehicles occupancy}

Passenger flow analysis can be made on the basis of the data from the bus occupancy indicator. These measurements belong to the most frequently performed measurements. They enable the assessment of transport efficiency of individual lines. The measurement can be carried out in many ways, which depend on the vehicle's capacity, its average occupancy and the size of the passenger exchange. The methods that can be used at low vehicle occupancy surveys are [Tracz, 1984]:

- counting passengers;

- counting free seats and subtracting the obtained number from the number of seats by getting the occupancy rate;

- counting standing passengers and adding them to the number of seats.

In the case of greater occupancy of vehicles during rush hours, the measurement is usually carried out on the outside of vehicles using the "photographic" method. Based on the model photos, the occupancy of the bus is estimated. This method does not provide too much accuracy, but it is simple to implement. The assessment of vehicle occupancy is also carried 
out by assessing the visual occupancy status of buses, distinguishing according to the WBR (Warsaw Traffic Research 2005) five basic types of buses, among others: short, medium-sized, long, articulated etc. [AECOM, 2009], where we distinguish 5 basic bus occupancy stages. There are also carried out surveys at bus stops, which not only have to show the buses'/trams' occupancy but are focused on the identification of transport behaviour of the residents and visitors. Other methods include the estimation of bus occupancy rates based on revenue from ticket sales or monitoring analysis.

The modes of public transport also use automatic counting of passengers. These methods use the stereoscopic image analysis technology [Infotron], directional sensors located under the vehicle's door, or sensors above the doors that emit a downstream infrared beam [Pixel] and vision detection systems [Łukasik Z., Kuśmińska-Fijałkowska A, Żurek-Mordka M., 2016; Zakład elektroniczny Letronik]. These equipments record all passenger exits and entrances through each of the doors of the vehicle: continuously, for each stop, throughout the period of work on the public transport line. These systems also register all passenger exits and entrances when the bus stops at a stop with the engine turned off. Passenger weighing systems [Mitas W.A., Bernaś M., Bugdol M, Ryguła A., Konior W., 2011; Ryguła A., Loga W., Brzozowski K., 2015] are increasingly used to measure passenger flows in public transport.

The measurement of the bus occupancy is usually performed together with the measurement of the passenger exchange rate, i.e. the number of people getting on and off at stops. Such a measurement can be carried out counting persons getting on and off at a bus stop and registering the arrival and departure time for calculating the time of passenger exchange, and the results obtained should be recorded in a suitably prepared form. For more lines and passengers, and if more accuracy is required, you can use the photographic method or frame-by-frame.

As a result of the measurement of the occupancy and the number of passengers getting on and off a mode of transportation, you can get the values of passenger flows on a given line. Based on the vehicle occupancy estimate (\%), after taking into account the size of a bus, the number of people traveling there is determined.

More accurate measurements of bus stop times at bus stops are required in some cases. Then the exact times are measured:

- arrival of the bus;

- opening the door;

- end of passenger exchange;

- closing the door;

- moving off;

- joining the traffic.

The described measurements require proper preparation in such things as [Tracz, 1984]:

- stablishing the location of stops with the numbers of lines on which vehicles stop and their frequency of running; 
- establishing traffic data for a given route;

- drawing up appropriate situational plans.

In the case of analyzing the advisability and effectiveness of the introduction of priorities for public transport, research must be expanded and should embrace all users of the public transport system [Tracz, 1984].

The analysis of the results of the study of traffic conditions and the quality of passenger transport services is carried out using statistical methods [Tracz, 1984].

It is worth paying attention to the fact that in the public transport services there is no clear criteria for the classification of the quality of this service.

Analyzes of the use of public transport in Radom were made on the basis of the research carried out by the authors as part of the project "Analysis of traffic and commuting preferences in the area of ROF", carried out as part of the project "Strategy for Urban Development of the Radom Function Area (ROF)" co-financed from the European Union Regional Development Fund and the state budget from the Operational Programme Technical Assistance 2007-2013, Radom June-August 2014. The aim of the project was to perform diagnostic research including: research into the traffic at selected points (over 100) in Radom, a vehicle occupancy survey and surveys generators of movement and the execution of the survey in the households of the inhabitants of the ROF, to determine the motivation and intensity of the movement of the ROF citizens and within the ROF. The article uses the results of the surveys concerning bus occupancy in public transport and the results of surveys in the households of the inhabitants of Radom - in this case, part of the survey, the so-called "journey diary", in which the respondents indicated all journeys made on the weekday preceding the research indicating, among others, their source-goal (destination) and means of transport.

\section{Vehicle occupancy surveys in public transport at selected final stops in Radom}

There were 25 bus lines in Radom in 2014. ${ }^{1}$ The lines operating within the city are divided into four categories: priority, basic, complementary and marginal lines. The priority lines include two lines: 7 (connecting the Michałów estate located northwards with the "Południe" estate) and 9 (running from the north-eastern Golębiów I estate to the Prędocinek estate located in the south), operating at peak times with a frequency of not less than 10 minutes. These lines run through the city centre and close to the railway and bus junctions, providing access to the four largest housing estates. The second group of the lines are basic lines (12 lines) running at their peak frequency every 15 minutes. The remaining lines are designed to complement the transport needs of people moving around in Radom. Their frequency at the rush hour is more than 20 minutes. The operators used 186 buses to service the public

1 The same number of lines operates in 2018. 
transport lines in Radom. Buses in the public transport in Radom transport about 40 million passengers per year.

An analysis of the routes of individual public transport lines in Radom was carried out, before starting the survey on selected public transport stops. The purpose of this analysis was to indicate the end stops at which surveys will be carried out with public transport drivers in Radom, so that each bus line will be tested in each direction of travel. 15 measurement points were chosen (Figure 1), in which questionnaire surveys with drivers were carried out.

\section{Figure 1. Location of measurement points for questionnaire surveys with drivers at end stops in Radom}

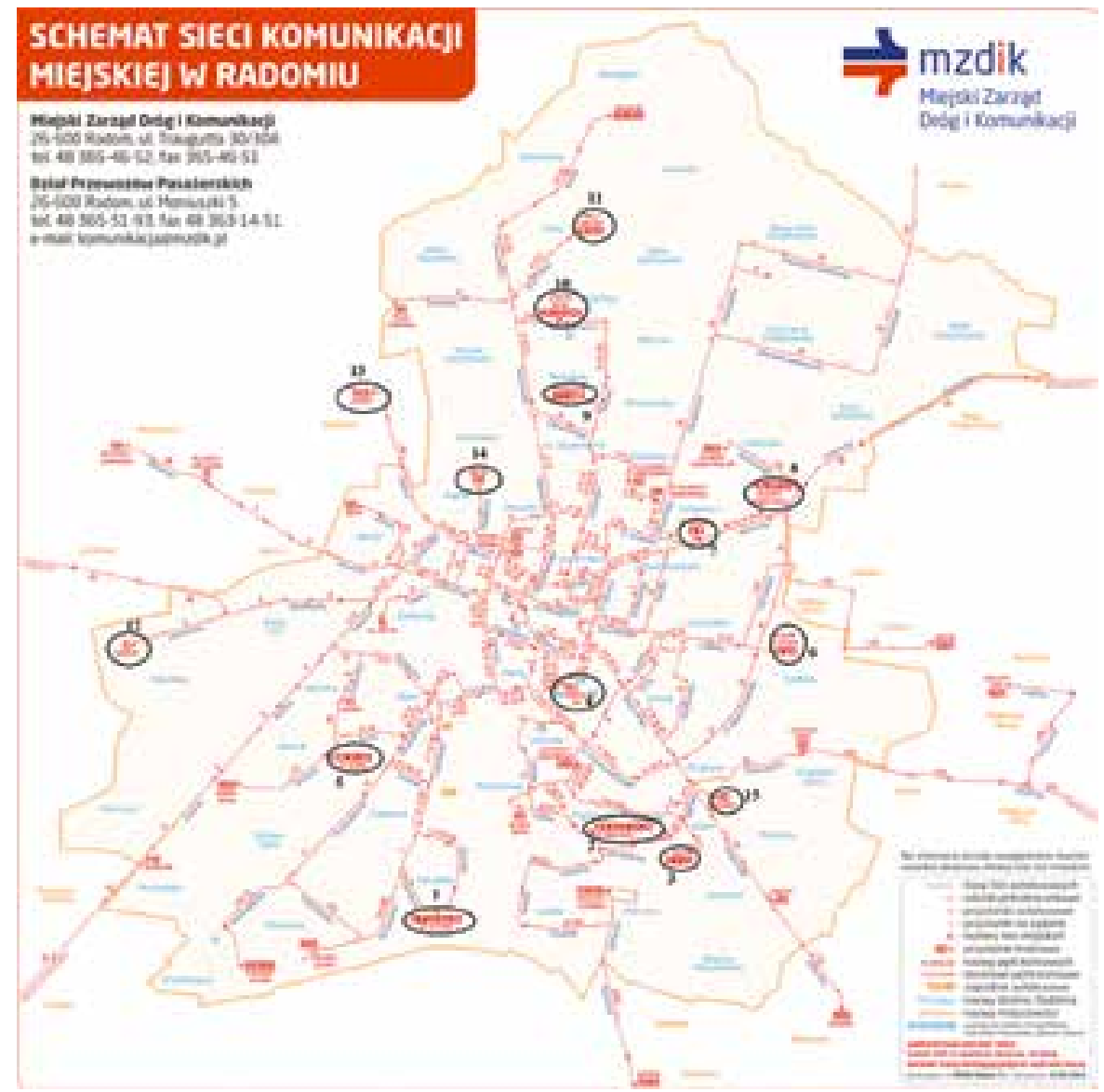

Source: Own study based on the scheme of the public transport network in Radom taken from the website of MZDiK in Radom. Retrieved from: http://www.mzdik.radom.pl/index.php?id=193 [accessed: 6.6.2014].

The survey research was carried out on June 4,2014. The interviewers collected information from the drivers about inflows in the means of transport of individual bus lines at certain times. The driver registered the occupancy of the means of transport in previously prepared forms during the journey at each stop. The form contained information on the estimated occupancy rating (\%) of the means of transport on the line operated by a given driver during 
the working day. It should be emphasized that in the literature one has not found publications that would present a method of measuring the occupancy in means of urban transport by conducting a survey with the drivers of these means of transport.

The results of the survey on a given route and at particular stops of a given line are presented in Tables 1-2. The analyzed data sets for each line were the average bus occupancy and average number of passengers on the bus on a given route (as the average at individual stops) and the average bus occupancy and average number of passengers on the bus at the bus stop (as the average from individual buses). Figures $2-5$ present examples of the average occupancy on a given route and the average occupancy at individual stops on two lines (numbers 7 and 11). ${ }^{2}$

The average daily vehicle occupancy indicator in the public transport in Radom, both in the aspect of inflating on particular routes of the line (Table 1) and at individual stops (Table 2) ranges from $19.8 \%$ to $59 \%$ and the mean is about $41 \%$ while the median is 40.3 in the aspect of inflating on particular routes of the line and 41.7 at individual stops. The remaining statistical measures are identical (0.1 percentage point difference) and amount to: $\mathrm{SD}=8.4$, Q1 = 35.1, Q3 = 46.8, although the occupancy at stops for individual lines shows a slightly higher variation (measured by the standard deviation and coefficient of variation) than the occupancy on the routes of the line. Only in 4 cases (on the 47 lines considered) the occupancy exceeds $50 \%$ (7b, 12a, 18 and 24a), and in 5 it does not exceed 30\% (2a, 2b, 5b, 19a, 19b).

\subsection{Vehicle occupancy surveys on the routes of individual lines}

The highest occupancy occurs on the lines No. $7 \mathrm{~b}$ and $24 \mathrm{a}$, with the median for the line $7 \mathrm{a}$ being slightly ( 0.8 percentage points) higher than the average, and in the case of the 24 a line 6 percentage points lower and $50 \%$. For line $7 \mathrm{~b}$, for $75 \%$ of the rates the average occupancy is not less than $50.6 \%$ and the maximum $86.8 \%$; the average number of passengers is 81.6 with a nearly $30 \%$ coefficient of variation ( \pm 23.9 passengers). For line $24 \mathrm{a}$, for $75 \%$ of the rates, the occupancy exceeds $44.7 \%$ and the maximum $100 \%$, which means that the whole bus took the maximum number of passengers throughout the route. The average number of passengers is 62 with the coefficient of variation equal to $36.7 \%$ ( \pm 22.8 passengers). The difference in the average number of passengers does not result from a significant difference in the occupancy but from the average capacity of buses ( 139 for lines $7 \mathrm{~b}$ and 115 for lines $24 \mathrm{a}$, for this line the largest and smallest occupancy "implemented" was a bus with a nominal capacity of 100 people). Please note that the average nominal capacity of public transport buses in Radom is 109 , minimum 86 , maximum 151 . The $12 \mathrm{a}$ line has the average occupancy of $52 \%$, the average

2 The data from the tables and drawings do not allow for a full analysis, in particular of individual routes or stops in a timely manner. For this it is necessary to analyze the occupancy at individual stops for individual routes. However, the data for one line takes as much space as tables 1 and 2 together. Therefore, it was decided to "compress the data" from a given line/line stop to the average value, i.e. the average occupancy in the route, the average occupancy at the stop of the given line and, respectively, the average number of passengers. For the average sets, the basic statistical parameters given in the tables were calculated. Unfortunately, the results obtained in the aspect of passenger exchange at stops did not allow analysing this issue. 
of 79 passengers and the average bus capacity of 151 and line 18 (a circular line) for which the respective values are 52\%, 50.8 and 99.6.

There are significant differences between lines $7 \mathrm{a}$ and $7 \mathrm{~b}$ and $24 \mathrm{a}$ and $24 \mathrm{~b}$. On line $7 \mathrm{a}$ (following the same route but in the opposite direction of every $7 \mathrm{~b}$ ), the occupancy level was much lower and amounted to $45.1 \%$ with the maximum of $57.9 \%$ (only $25 \%$ of the trips had the occupancy of at least 50\%), and the average number of travelers was 66.7 ( \pm 12.5 passengers). The average nominal capacity was 147.3 . For line $24 \mathrm{~b}$ the average occupancy was $38.5 \%$. (minimum $23.7 \%$, maximum 60.7\%), the average number of passengers 40.7 ( \pm 11.3 passengers) and the average nominal capacity 107.9 (i.e. lower than for 24a). Only for these two lines we can see such significant differences between the "there" and "back" routes, and for almost all the measured parameters. For the remaining lines, these differences are much smaller or insignificant and concern only some parameters (the variation coefficient for lines 14, 15, 26, minimum occupancy for lines 13, 14, 26, Q1 for lines 10 and 26, maximum occupancy for lines 5 , the coefficient of variation for the number of passengers for lines 14, 15, 26). For 11 lines (out of 47), the average occupancy range is not less than $60 \mathrm{pp}$, and for 28 the median is larger than the mean value (in one case it is equal to the mean value).

The lines number 7 and 9 are priority lines. For lines $9 a$ and $9 b$, the average occupancy is slightly higher than $47 \%$ with a range from $20 \%$ to $80 \%$, the average number of passengers is 53.4 and 54.9, respectively, and the capacities 113 and 116.4, respectively. The differences between lines $9 \mathrm{a}$ and $9 \mathrm{~b}$ are small, in contrast to lines $7 \mathrm{a}$ and $7 \mathrm{~b}$.

Table 1. The vehicle occupancy indicator on the routes of individual lines*

\begin{tabular}{|c|l|r|r|r|r|r|c|c|c|c|c|c|c|c|}
\hline NL & \multicolumn{1}{|c|}{$P$} & MBC & \multicolumn{1}{|c|}{ SD } & \multicolumn{1}{c|}{ CV } & Me & Min & Q1 & Q3 & Max & MC & SD & CV & NC & MBN \\
\hline 1a & Południe & 43.8 & 7.1 & 16.2 & 44.3 & 27.3 & 38.9 & 49.6 & 57.3 & 43.8 & 7.1 & 16.2 & 40 & 100.0 \\
\hline 1b & Gołębiów & 47.3 & 11.0 & 23.2 & 47.8 & 28.3 & 37.0 & 56.1 & 71.3 & 47.3 & 11.0 & 23.2 & 41 & 100.0 \\
\hline 2a & Zamłynie & $\mathbf{2 8 . 7}$ & $\mathbf{1 2 . 4}$ & $\mathbf{4 3 . 3}$ & $\mathbf{2 5 . 3}$ & $\mathbf{1 4 . 3}$ & $\mathbf{1 9 . 0}$ & $\mathbf{3 4 . 9}$ & $\mathbf{5 7 . 5}$ & $\mathbf{2 9 . 1}$ & $\mathbf{1 2 . 6}$ & $\mathbf{4 3 . 2}$ & $\mathbf{2 9}$ & $\mathbf{1 0 1 . 6}$ \\
\hline 2b & Idalin & $\mathbf{2 6 . 1}$ & $\mathbf{1 0 . 4}$ & $\mathbf{3 9 . 8}$ & $\mathbf{2 4 . 2}$ & $\mathbf{1 1 . 9}$ & $\mathbf{1 8 . 5}$ & $\mathbf{3 1 . 0}$ & $\mathbf{5 9 . 0}$ & $\mathbf{2 6 . 4}$ & $\mathbf{1 0 . 9}$ & $\mathbf{4 1 . 3}$ & $\mathbf{3 0}$ & $\mathbf{1 0 0 . 7}$ \\
\hline 3a & Michałów & 43.5 & 13.4 & 30.9 & 40.4 & 20.6 & 34.0 & 48.8 & 80.6 & 37.5 & 11.3 & 30.1 & 37 & 86.6 \\
\hline 3b & Idalin & 49.5 & 12.9 & 26.0 & 50.0 & 20.4 & 39.8 & 58.5 & 73.3 & 46.7 & 12.9 & 27.6 & 37 & 94.6 \\
\hline 4a & Prędocinek & 37.2 & 10.7 & 28.8 & 36.7 & 18.8 & 29.5 & 44.1 & 66.7 & 48.2 & 15.3 & 31.7 & 36 & 128.9 \\
\hline 4b & Firlej Cmentarz & 34.4 & 8.1 & 23.7 & 34.6 & 19.2 & 29.2 & 38.3 & 54.2 & 44.6 & 12.0 & 26.9 & 41 & 129.3 \\
\hline 5a & Sadków/Lotnisko & 33.3 & 13.4 & 40.2 & 32.4 & 13.8 & 22.3 & 39.6 & 83.6 & 31.6 & 12.7 & 40.2 & 42 & 95.0 \\
\hline 5b & Pruszaków/Młodocin & 27.8 & 12.6 & 45.4 & 26.7 & 9.6 & 18.9 & 33.5 & 55.9 & 26.4 & 12.0 & 45.4 & 37 & 95.0 \\
\hline 6a & Prędocinek & 40.8 & 10.1 & 24.7 & 42.1 & 22.9 & 36.8 & 47.1 & 59.3 & 38.7 & 9.5 & 24.7 & 25 & 95.0 \\
\hline 6b & Milejowice & 44.0 & 8.5 & 19.2 & 43.7 & 26.3 & 40.3 & 49.3 & 58.3 & 41.8 & 8.0 & 19.2 & 29 & 95.0 \\
\hline 7a & Południe & 45.1 & 6.4 & 14.2 & 45.2 & 28.6 & 41.3 & 50.0 & 57.9 & 66.7 & 12.5 & 18.8 & 75 & 147.3 \\
\hline 7b & Michałów & $\mathbf{5 8 . 9}$ & $\mathbf{1 3 . 7}$ & $\mathbf{2 3 . 2}$ & $\mathbf{5 9 . 8}$ & $\mathbf{2 3 . 2}$ & $\mathbf{5 0 . 6}$ & $\mathbf{6 7 . 5}$ & $\mathbf{8 6 . 8}$ & $\mathbf{8 1 . 6}$ & $\mathbf{2 3 . 9}$ & $\mathbf{2 9 . 3}$ & $\mathbf{6 6}$ & $\mathbf{1 3 9 . 1}$ \\
\hline 8a & Wośniki & 43.4 & 8.7 & 20.0 & 42.8 & 23.6 & 37.9 & 50.8 & 56.0 & 43.4 & 8.7 & 20.0 & 17 & 100.0 \\
\hline 8b & Kierzków & 44.1 & 8.5 & 19.3 & 46.9 & 21.5 & 40.6 & 49.6 & 53.5 & 44.1 & 8.5 & 19.3 & 15 & 100.0 \\
\hline 9a & Gołębiów & 47.3 & 15.3 & 32.3 & 45.8 & 20.0 & 34.4 & 61.3 & 80.0 & 53.4 & 19.1 & 35.7 & 74 & 113.0 \\
\hline 9b & Prędocinek & 47.1 & 11.8 & 25.1 & 48.1 & 19.7 & 37.8 & 55.0 & 74.4 & 54.9 & 14.8 & 26.9 & 73 & 116.4 \\
\hline
\end{tabular}




\begin{tabular}{|c|c|c|c|c|c|c|c|c|c|c|c|c|c|c|}
\hline $\mathrm{NL}$ & $P$ & MBC & SD & CV & $\mathrm{Me}$ & Min & Q1 & Q3 & $\operatorname{Max}$ & MC & SD & CV & NC & MBN \\
\hline $10 \mathrm{a}$ & Wacyn & 39.6 & 10.3 & 26.0 & 39.0 & 24.5 & 30.7 & 46.0 & 65.5 & 38.5 & 9.9 & 25.6 & 20 & 97.5 \\
\hline $10 \mathrm{~b}$ & $\begin{array}{l}\text { Gołębiów/Rodziny } \\
\text { Ziętalów }\end{array}$ & 44.5 & 10.9 & 24.5 & 42.5 & 28.8 & 39.6 & 48.3 & 70.0 & 41.8 & 9.6 & 23.0 & 9 & 94.6 \\
\hline $11 a$ & Idalin & 36.4 & 13.7 & 37.5 & 33.3 & 14.2 & 24.5 & 46.6 & 63.5 & 35.0 & 12.2 & 34.9 & 36 & 97.2 \\
\hline $11 b$ & $\begin{array}{l}\text { Gołębiów/ } \\
\text { Paderewskiego }\end{array}$ & 35.5 & 11.6 & 32.8 & 33.6 & 19.5 & 26.4 & 42.7 & 61.8 & 34.0 & 11.0 & 32.2 & 33 & 96.5 \\
\hline $12 \mathrm{a}$ & Południe & 52.3 & 5.8 & 11.0 & 52.6 & 38.0 & 49.8 & 55.9 & 65.2 & 79.2 & 12.8 & 16.1 & 27 & 151.0 \\
\hline $12 \mathrm{~b}$ & Wincentów & 49.6 & 3.8 & 7.6 & 50.3 & 40.3 & 47.0 & 52.3 & 54.8 & 73.9 & 10.2 & 13.8 & 29 & 148.8 \\
\hline $13 a$ & Józefów Szpital & 46.5 & 11.8 & 25.4 & 46.0 & 28.4 & 37.5 & 52.2 & 75.6 & 50.7 & 23.2 & 45.8 & 36 & 107.6 \\
\hline $13 b$ & Wośniki Szkoła & 48.1 & 13.0 & 27.0 & 47.6 & 16.7 & 41.2 & 54.2 & 89.6 & 53.4 & 21.6 & 40.5 & 28 & 110.2 \\
\hline $14 a$ & Sadków/Lotnisko & 35.9 & 14.5 & 40.5 & 35.5 & 8.0 & 23.9 & 45.0 & 74.4 & 33.9 & 15.1 & 44.7 & 34 & 93.8 \\
\hline $14 b$ & Południe & 39.6 & 8.4 & 21.1 & 41.1 & 20.8 & 34.9 & 45.8 & 56.6 & 38.1 & 9.7 & 25.5 & 34 & 95.3 \\
\hline $15 \mathrm{a}$ & Kaptur & 46.1 & 19.1 & 41.5 & 42.0 & 15.0 & 30.0 & 65.0 & 81.4 & 40.7 & 18.0 & 44.3 & 37 & 87.6 \\
\hline $15 b$ & Janiszpol & 48.8 & 14.4 & 29.6 & 50.0 & 20.0 & 40.0 & 60.0 & 75.0 & 42.1 & 13.6 & 32.2 & 33 & 86.1 \\
\hline $16 a$ & $\begin{array}{l}\text { Gołębiów II/ } \\
\text { Sempołowskiej }\end{array}$ & 48.4 & 9.9 & 20.5 & 48.4 & 30.0 & 41.8 & 56.4 & 68.2 & 48.4 & 9.9 & 20.5 & 18 & 100.0 \\
\hline $16 b$ & Wośniki & 46.4 & 8.9 & 19.2 & 42.5 & 34.8 & 40.1 & 54.4 & 62.7 & 46.4 & 8.9 & 19.2 & 14 & 100.0 \\
\hline $17 \mathrm{a}$ & Potkanów/Salowa & 43.3 & 15.2 & 35.1 & 43.2 & 10.9 & 34.5 & 51.4 & 71.2 & 63.7 & 26.0 & 40.7 & 41 & 145.5 \\
\hline $17 b$ & Gołębiów/Zubrzyckiego & 45.8 & 16.7 & 36.5 & 45.2 & 7.5 & 35.2 & 51.4 & 84.3 & 68.6 & 28.7 & 41.8 & 37 & 148.9 \\
\hline 18 & Dworzec PKP** & 52.0 & 12.0 & 23.1 & 54.2 & 20.3 & 44.6 & 61.4 & 70.6 & 50.8 & 11.0 & 21.6 & 28 & 99.6 \\
\hline $19 a$ & Obozisko & 21.6 & 9.8 & 45.3 & 26.0 & 2.3 & 13.2 & 29.6 & 35.5 & 22.9 & 10.4 & 45.3 & 22 & 106.0 \\
\hline $19 b$ & Malenice & 19.8 & 9.4 & 47.5 & 18.8 & 4.7 & 14.8 & 26.7 & 44.9 & 21.0 & 10.0 & 47.5 & 19 & 106.0 \\
\hline $21 \mathrm{a}$ & Prędocinek & 34.7 & 6.0 & 17.3 & 35.2 & 19.7 & 31.9 & 38.1 & 47.1 & 37.5 & 7.2 & 19.2 & 21 & 107.9 \\
\hline $21 b$ & Wólka Klwatecka & 36.8 & 7.2 & 19.5 & 39.0 & 22.3 & 33.0 & 41.3 & 46.7 & 41.0 & 9.1 & 22.1 & 19 & 111.3 \\
\hline 23a & Józefów Szpital & 30.1 & 16.2 & 53.6 & 24.6 & 5.4 & 17.0 & 41.4 & 65.6 & 33.4 & 16.6 & 49.7 & 37 & 117.0 \\
\hline $23 b$ & Prędocinek & 34.2 & 16.8 & 49.1 & 30.8 & 11.8 & 21.8 & 44.7 & 72.8 & 41.0 & 20.5 & 49.9 & 24 & 124.1 \\
\hline $24 a$ & Michałów & 56.0 & 22.1 & 39.5 & 50.0 & 19.5 & 44.7 & 71.0 & 100.0 & 62.0 & 22.8 & 36.7 & 11 & 115.0 \\
\hline $24 b$ & Małęczyn Nowy & 38.5 & 10.4 & 27.2 & 36.5 & 23.7 & 29.8 & 45.1 & 60.7 & 40.7 & 11.3 & 27.7 & 12 & 107.9 \\
\hline $25 a$ & Prędocinek & 34.9 & 8.3 & 23.8 & 33.3 & 20.2 & 29.0 & 42.6 & 48.6 & 42.5 & 10.6 & 24.9 & 23 & 121.5 \\
\hline $25 b$ & Potkanów/Żelazna & 33.8 & 8.4 & 24.8 & 33.2 & 16.5 & 29.1 & 37.4 & 47.6 & 32.0 & 17.9 & 55.9 & 21 & 122.4 \\
\hline $26 \mathrm{a}$ & Myśliszewice & 39.5 & 17.8 & 45.1 & 41.1 & 8.1 & 24.8 & 53.0 & 71.9 & 38.0 & 17.1 & 45.1 & 23 & 96.0 \\
\hline \multirow[t]{4}{*}{$26 \mathrm{~b}$} & Janiszew & 44.1 & 13.1 & 29.6 & 43.4 & 25.6 & 33.4 & 52.9 & 67.8 & 42.4 & 12.5 & 29.6 & 23 & 96.0 \\
\hline & Mean & 41.0 & 11.5 & 29.3 & 40.3 & 19.7 & 33.0 & 48.5 & 65.6 & 44.7 & 13.6 & 31.8 & 31.8 & 109.1 \\
\hline & Min & 19.8 & 3.8 & 7.6 & 18.8 & 2.3 & 13.2 & 26.7 & 35.5 & 21.0 & 7.1 & 13.8 & 9.0 & 86.1 \\
\hline & Max & 58.9 & 22.1 & 53.6 & 59.8 & 40.3 & 50.6 & 71.0 & 100.0 & 81.6 & 28.7 & 55.9 & 75.0 & 151.0 \\
\hline
\end{tabular}

NL - line number; $\mathrm{P}$ - the final bus stop (identifies the direction of bus traffic on the line); $\mathrm{MBC}$ - average daily vehicle occupancy indicator [\%]; SD - standard deviation [percentage point]; CV - coefficient of variation [\%]; Me - median [\%]; Q1 - first quartile [\%]; Q3 - third quartile [\%]; MC - average daily number of passengers on the bus on each line [number of people]; NC - Number of trips (for which the measurement data was available, for some of the trips there was no full data e.g. due to a bus failure or refusal to complete the questionnaire by the bus driver); $\mathrm{MBN}$ - average nominal vehicle capacity [number of people].

${ }^{*}$ the set examined was a set of average bus occupancy (in percent) on the routes of individual lines.

${ }^{* *}$ the 18 line is a line that goes in a circular manner.

Source: Own material. 
Some of the smallest differences between the "there" and "back" routes are observed for lines $19 \mathrm{a}$ and $19 \mathrm{~b}$ and $2 \mathrm{a}$ and $2 \mathrm{~b}$, for which we observe the lowest average occupancy. For line $19 \mathrm{a} 21.6 \%$ ( $\pm 9.8 \mathrm{pp})$ and for line $19 \mathrm{~b} 19.8 \%$ ( $\pm 9.4 \mathrm{pp})$ with the coefficient of volatility exceeding $45 \%$. The average daily number of passengers on the bus was 22.9 persons for lines $19 \mathrm{a}$ and 21 for the $19 \mathrm{~b}$ line with the average bus capacity 106. Also for line 2 there was a low level of average occupancy of $2 \mathrm{a}$ line $-28.7 \%$ ( $\pm 12.4 \mathrm{pp}, \mathrm{CV}=43.3 \%)$, for line $2 \mathrm{~b}-26.1 \%$ $( \pm 10.4 \mathrm{pp}, \mathrm{CV}=39.8 \%)$ with the average number of passengers $29.1(\mathrm{CV}=43.2 \%)$ and 26.4, respectively. ( $\mathrm{CV}=41.3 \%)$ and the average bus capacities of 101.6 and 100.7 .

Figures 2 and 3 present examples of the average bus occupancy schedules for individual routes of lines 7 and 11. These charts show the variation of average occupancy rates at individual line routes, which together with the average occupancy of a given line at the stops give a fuller picture of the variability of occupancy (Fig. 4 and 5). ${ }^{3}$ Line $7 \mathrm{~b}$ has the largest average occupancy of all the lines, however, compared to line $7 \mathrm{a}$ it has much greater variability of individual routes (the difference in stretch marks is almost $31 \%$ with the standard deviation over 7 pp: Table 1). Particularly large variations can be observed in the morning trips. Bus line $7 \mathrm{~b}$ is the main transport line to the railway and bus stations and the city centre for people living in the South. Line 11 belongs to the line with a lower vehicle occupancy (below the average, at the level of $35.5 \%$ ) despite a relatively small capacity of buses serving it (about 97). It is also a line for which there are no significant differences between the "there" and "back" rates.

Figure 2. Average occupancy of buses on individual line routes: $a-N o .7 a, b-N o .7 b$

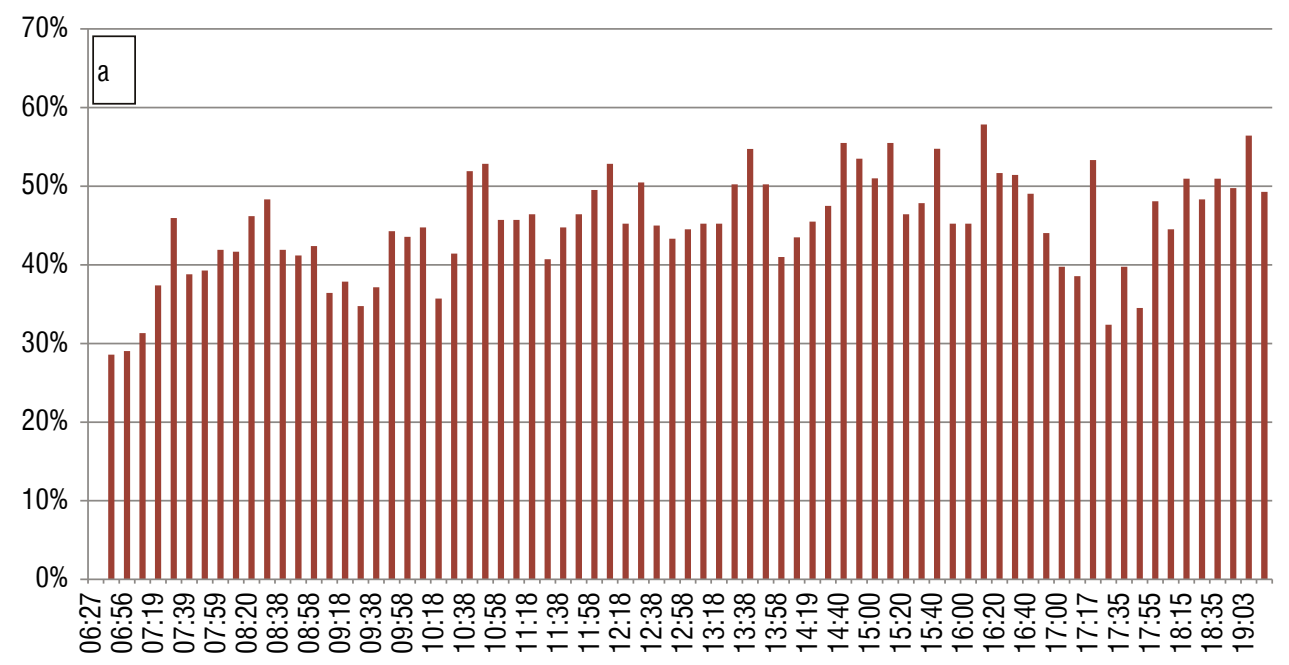

3 Although further, due to the medium operation and the lack of information and bus capacity on the route, they do not give a full picture of the variability of capacity during the bus's trip. They also do not provide information on the exchange of passengers, and therefore the number of passengers transported. 


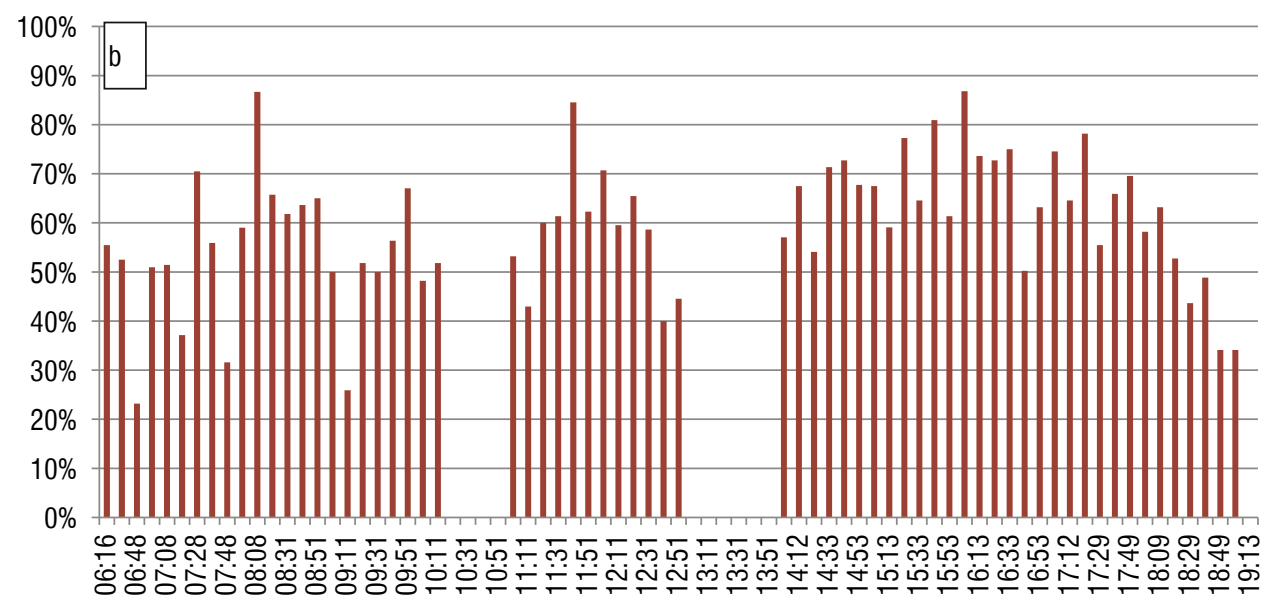

Source: Own material.

Figure 3. Average occupancy of buses on individual line routes: $a-$ No. $11 a, b-$ No. $11 b$
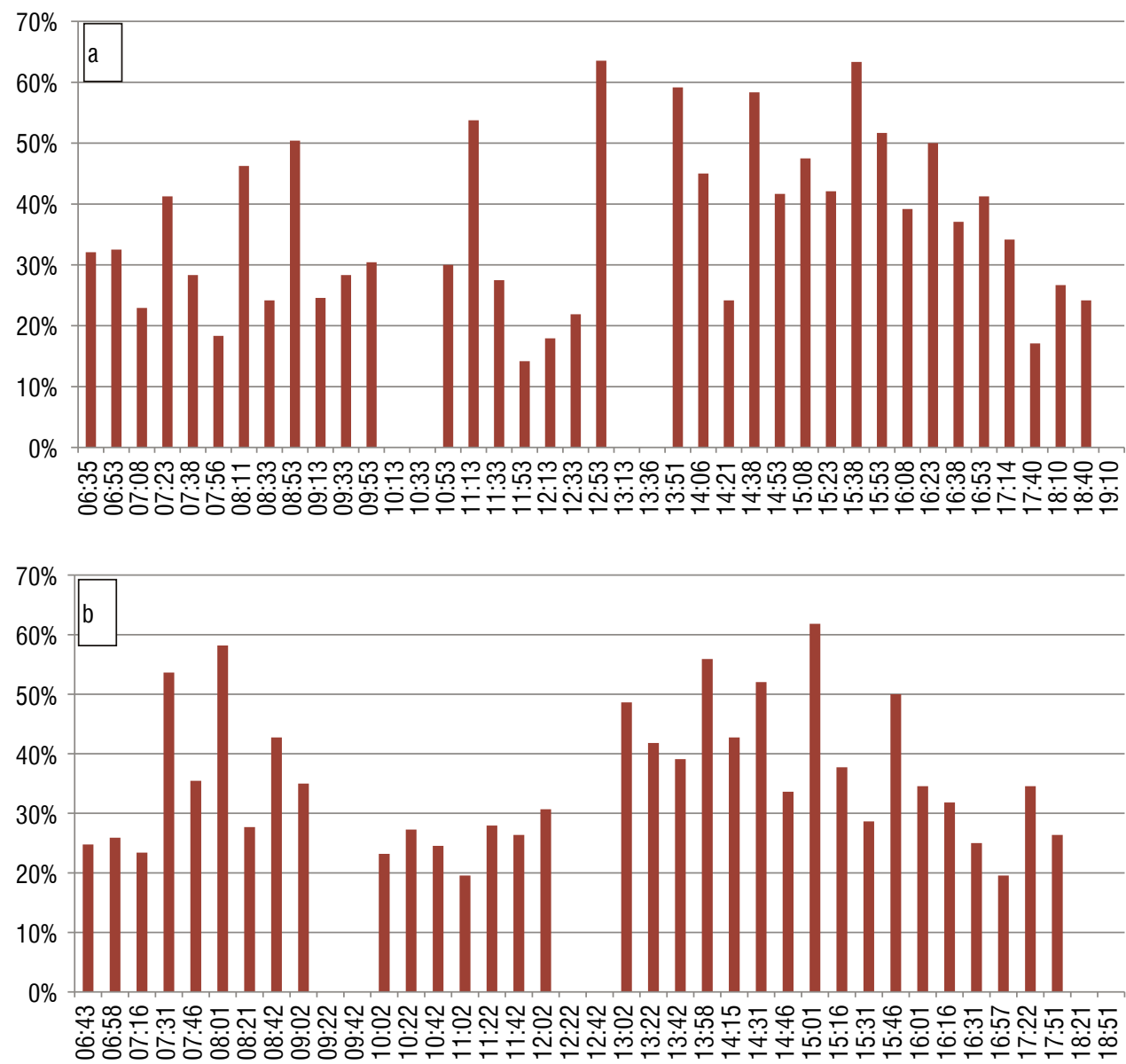

Source: Own material. 


\subsection{Vehicle occupancy indicator at the bus stops of individual lines}

Table 2. Bus occupancy at the bus stops of individual lines*

\begin{tabular}{|c|c|c|c|c|c|c|c|c|c|c|c|c|c|c|}
\hline $\mathrm{NL}$ & $P$ & MBP & SD & CV & $\mathrm{Me}$ & Min & Q1 & Q3 & Max & MP & SD & CV & NS & MBN \\
\hline $1 a$ & Południe & 43.8 & 13.3 & 30.2 & 46.0 & 22.4 & 30.1 & 56.3 & 60.4 & 43.8 & 13.3 & 30.2 & 22 & 100.0 \\
\hline $1 b$ & Gołębiów & 47.3 & 9.6 & 20.2 & 45.9 & 32.8 & 39.4 & 56.9 & 61.1 & 47.3 & 9.5 & 20.1 & 23 & 100.0 \\
\hline $2 a$ & Zamłynie & 28.7 & 5.9 & 20.7 & 30.7 & 20.3 & 21.8 & 34.1 & 36.6 & 29.1 & 6.0 & 20.5 & 20 & 101.6 \\
\hline $2 b$ & Idalin & 26.1 & 5.6 & 21.3 & 28.4 & 13.1 & 22.2 & 30.3 & 33.9 & 26.4 & 5.7 & 21.4 & 21 & 100.7 \\
\hline $3 a$ & Michałów & 43.0 & 9.8 & 22.9 & 43.5 & 25.3 & 39.8 & 51.4 & 56.1 & 37.2 & 8.4 & 22.7 & 26 & 86.6 \\
\hline $3 b$ & Idalin & 49.5 & 15.6 & 31.6 & 52.6 & 18.2 & 48.5 & 63.3 & 65.5 & 46.7 & 14.7 & 31.6 & 24 & 94.6 \\
\hline $4 a$ & Prędocinek & 37.2 & 17.6 & 47.4 & 38.9 & 10.7 & 25.2 & 56.0 & 61.9 & 48.2 & 22.8 & 47.3 & 24 & 128.9 \\
\hline $4 b$ & Firlej Cmentarz & 34.4 & 17.3 & 50.5 & 33.8 & 9.6 & 17.2 & 49.0 & 59.3 & 44.6 & 22.4 & 50.3 & 24 & 129.3 \\
\hline $5 a$ & Sadków/Lotnisko & 33.8 & 9.2 & 27.1 & 35.5 & 15.4 & 27.2 & 38.2 & 48.9 & 32.1 & 8.7 & 27.1 & 28 & 95.0 \\
\hline $5 b$ & Pruszaków/Młodocin & 27.3 & 7.5 & 27.4 & 25.4 & 16.2 & 20.8 & 33.7 & 39.3 & 25.9 & 7.1 & 27.4 & 27 & 95.0 \\
\hline $6 a$ & Prędocinek & 40.8 & 19.1 & 47.0 & 44.4 & 10.0 & 23.5 & 58.8 & 63.6 & 38.7 & 18.2 & 47.0 & 28 & 95.0 \\
\hline $6 b$ & Milejowice & 44.0 & 20.1 & 45.8 & 43.8 & 14.0 & 22.8 & 58.5 & 69.3 & 41.8 & 19.1 & 45.8 & 30 & 95.0 \\
\hline $7 a$ & Południe & 45.0 & 11.7 & 26.1 & 48.4 & 27.2 & 33.5 & 57.1 & 60.1 & 66.5 & 17.3 & 26.1 & 21 & 147.3 \\
\hline $7 \mathrm{~b}$ & Michałów & 59.0 & 8.5 & 14.4 & 60.8 & 39.2 & 56.9 & 62.5 & 68.5 & 81.6 & 11.9 & 14.6 & 22 & 139.1 \\
\hline $8 a$ & Wośniki & 43.1 & 14.0 & 32.6 & 41.3 & 27.1 & 28.5 & 60.0 & 64.1 & 43.0 & 14.1 & 32.8 & 25 & 100.0 \\
\hline $8 b$ & Kierzków & 44.1 & 15.6 & 35.4 & 44.3 & 24.3 & 28.9 & 60.8 & 66.3 & 44.1 & 15.6 & 35.4 & 26 & 100.0 \\
\hline $9 a$ & Gołębiów & 47.3 & 8.7 & 18.5 & 44.3 & 34.1 & 42.4 & 56.8 & 58.8 & 53.4 & 9.7 & 18.1 & 15 & 113.0 \\
\hline $9 b$ & Prędocinek & 47.1 & 11.1 & 23.5 & 45.4 & 32.1 & 38.1 & 57.1 & 61.9 & 54.9 & 12.8 & 23.3 & 16 & 116.4 \\
\hline $10 \mathrm{a}$ & Wacyn & 39.6 & 8.5 & 21.5 & 40.9 & 22.9 & 34.8 & 46.8 & 50.8 & 38.5 & 8.3 & 21.7 & 22 & 97.5 \\
\hline $10 b$ & $\begin{array}{l}\text { Gołębiów/Rodziny } \\
\text { Ziętalów }\end{array}$ & 44.5 & 7.2 & 16.1 & 42.2 & 33.3 & 40.0 & 53.3 & 54.4 & 41.8 & 6.8 & 16.4 & 24 & 94.6 \\
\hline $11 a$ & Idalin & 36.4 & 10.7 & 29.3 & 40.4 & 23.3 & 23.9 & 45.7 & 51.0 & 35.0 & 10.2 & 29.1 & 24 & 97.2 \\
\hline $11 b$ & $\begin{array}{l}\text { Gołębiów/ } \\
\text { Paderewskiego }\end{array}$ & 35.5 & 9.9 & 28.0 & 39.2 & 21.2 & 25.7 & 43.9 & 47.0 & 34.0 & 9.6 & 28.3 & & \\
\hline $12 a$ & Południe & 52.8 & 10.0 & 18.9 & 53.5 & 36.7 & 43.1 & 62.3 & 65.9 & 80.0 & 15.0 & 18.8 & 32 & 151.0 \\
\hline $12 b$ & Wincentów & 50.3 & 11.3 & 22.4 & 54.1 & 29.1 & 39.4 & 60.9 & 64.0 & 74.8 & 16.5 & 22.1 & 32 & 148.8 \\
\hline $13 a$ & Józefów Szpital & 46.4 & 8.2 & 17.7 & 46.5 & 34.5 & 39.0 & 55.6 & 57.4 & 50.4 & 8.9 & 17.6 & 27 & 107.6 \\
\hline $13 b$ & Wośniki Szkoła & 47.8 & 9.9 & 20.8 & 45.6 & 32.7 & 37.4 & 56.1 & 61.1 & 53.3 & 11.1 & 20.9 & 25 & 110.2 \\
\hline $14 a$ & Sadków/Lotnisko & 35.5 & 10.1 & 28.3 & 30.0 & 13.1 & 29.5 & 43.5 & 53.2 & 33.6 & 9.6 & 28.6 & 32 & 93.8 \\
\hline $14 b$ & Południe & 37.3 & 7.3 & 19.6 & 36.8 & 24.0 & 33.7 & 43.0 & 48.8 & 35.8 & 7.0 & 19.5 & 32 & 95.3 \\
\hline $15 a$ & Kaptur & 46.1 & 4.2 & 9.1 & 49.1 & 38.7 & 40.9 & 50.1 & 50.1 & 40.7 & 3.6 & 8.9 & 21 & 87.6 \\
\hline $15 b$ & Janiszpol & 48.8 & 1.1 & 2.4 & 48.0 & 47.7 & 47.7 & 50.2 & 50.2 & 42.1 & 1.0 & 2.4 & 20 & 86.1 \\
\hline $16 a$ & $\begin{array}{l}\text { Gołębiów II/ } \\
\text { Sempołowskiej }\end{array}$ & 48.4 & 9.6 & 19.8 & 50.0 & 32.2 & 41.1 & 57.2 & 61.7 & 48.4 & 9.6 & 19.8 & 22 & 100.0 \\
\hline $16 b$ & Wośniki & 46.4 & 7.3 & 15.7 & 46.4 & 36.1 & 41.1 & 53.1 & 57.1 & 46.4 & 7.3 & 15.7 & 22 & 100.0 \\
\hline $17 \mathrm{a}$ & Potkanów/Salowa & 43.3 & 10.3 & 23.7 & 46.3 & 5.0 & 35.2 & 51.2 & 53.3 & 63.7 & 15.1 & 23.7 & 30 & 145.5 \\
\hline $17 \mathrm{~b}$ & Gołębiów/Zubrzyckiego & 45.8 & 6.6 & 14.5 & 46.9 & 34.4 & 37.8 & 51.8 & 52.4 & 68.6 & 9.7 & 14.1 & 28 & 148.9 \\
\hline 18 & Dworzec PKP** & 49.4 & 15.2 & 30.7 & 56.4 & 18.6 & 39.1 & 60.4 & 70.7 & 48.2 & 15.1 & 31.2 & 35 & 99.6 \\
\hline $19 a$ & Obozisko & 21.6 & 5.0 & 23.2 & 21.9 & 10.5 & 20.5 & 25.4 & 29.1 & 22.9 & 5.3 & 23.2 & 15 & 106.0 \\
\hline
\end{tabular}




\begin{tabular}{|l|l|r|r|r|r|r|r|r|r|r|r|r|r|r|}
\hline NL & \multicolumn{1}{|c|}{$P$} & MBP & \multicolumn{1}{|c|}{ SD } & \multicolumn{1}{c|}{ CV } & \multicolumn{1}{|c|}{ Me } & \multicolumn{1}{|c|}{ Min } & Q1 & Q3 & Max & MP & SD & CV & NS & MBN \\
\hline 19b & Malenice & $\mathbf{1 9 . 8}$ & $\mathbf{4 . 5}$ & $\mathbf{2 2 . 9}$ & $\mathbf{2 1 . 6}$ & $\mathbf{9 . 1}$ & $\mathbf{1 8 . 2}$ & $\mathbf{2 3 . 2}$ & $\mathbf{2 4 . 5}$ & $\mathbf{2 1 . 0}$ & $\mathbf{4 . 9}$ & $\mathbf{2 3 . 3}$ & $\mathbf{1 6}$ & $\mathbf{1 0 6 . 0}$ \\
\hline 21a & Prędocinek & 34.7 & 17.6 & 50.9 & 40.5 & 11.9 & 18.2 & 51.4 & 59.0 & 37.5 & 19.1 & 50.8 & 31 & 107.9 \\
\hline 21b & Wólka Klwatecka & 36.8 & 18.2 & 49.5 & 36.3 & 8.9 & 24.1 & 54.2 & 64.2 & 41.0 & 20.3 & 49.6 & 30 & 111.3 \\
\hline 23a & Józefów Szpital & 30.1 & 8.5 & 28.2 & 31.0 & 17.8 & 22.5 & 37.8 & 41.4 & 33.4 & 9.0 & 26.8 & 25 & 117.0 \\
\hline 23b & Prędocinek & 34.2 & 7.1 & 20.8 & 37.1 & 17.6 & 30.0 & 40.2 & 41.5 & 41.0 & 8.2 & 19.9 & 25 & 124.1 \\
\hline 24a & Michałów & 54.9 & 6.1 & 11.1 & 57.0 & 39.0 & 52.9 & 59.1 & 63.2 & 62.1 & 7.6 & 12.2 & 30 & 115.0 \\
\hline 24b & Małęczyn Nowy & 37.0 & 8.1 & 21.8 & 38.3 & 24.3 & 33.3 & 45.0 & 46.7 & 39.3 & 9.0 & 22.8 & 29 & 107.9 \\
\hline 25a & Prędocinek & 31.6 & 16.5 & 52.1 & 29.6 & 5.0 & 19.3 & 49.8 & 53.7 & 38.4 & 20.0 & 52.2 & 25 & 121.5 \\
\hline 25b & Potkanów/Żelazna & 31.0 & 17.3 & 55.8 & 26.2 & 12.9 & 15.7 & 45.2 & 58.6 & 30.2 & 15.6 & 51.7 & 23 & 122.4 \\
\hline 26a & Myśliszewice & 39.5 & 11.5 & 29.2 & 43.7 & 25.0 & 28.6 & 49.2 & 56.7 & 38.0 & 11.1 & 29.2 & 27 & 96.0 \\
\hline 26b & Janiszew & 43.1 & 10.7 & 24.8 & 51.7 & 26.4 & 35.2 & 52.3 & 53.3 & 41.4 & 10.3 & 24.8 & 27 & 96.0 \\
\hline & Mean & 40.6 & 10.6 & 27.1 & 41.7 & 23.1 & 32.2 & 50.2 & 54.8 & 44.4 & 11.5 & 27.0 & 25.0 & 109.1 \\
\hline & Min & 19.8 & 1.1 & 2.4 & 21.6 & 5.0 & 15.7 & 23.2 & 24.5 & 21.0 & 1.0 & 2.4 & 15.0 & 86.1 \\
\hline & Max & 59.0 & 20.1 & 55.8 & 60.8 & 47.7 & 56.9 & 63.3 & 70.7 & 81.6 & 22.8 & 52.2 & 35.0 & 151.0 \\
\hline
\end{tabular}

$\mathrm{NL}$ - line number; P - the final bus stop (identifies the direction of the bus traffic on the line); MBP - the average daily vehicle occupancy indicator at the bus stop of each bus line [\%]; SD - standard deviation [percentage point]; CV - coefficient of variation [\%]; Me - median [\%]; Q1 - first quartile [\%]; Q3 - third quartile [\%]; MP - the average daily number of passengers at bus stops on each bus line [number of people]; NS - Number of stops (it is the maximum number of line stops, for some routes it may be smaller - shortened/extended route, exit to the base, departure from the base); MBN - the average nominal vehicle capacity [number of people].

* the set checked was a set of the average bus occupancy (in percent) of the lines at individual bus stops.

${ }^{* *}$ the 18 line is a line that goes in a circular manner.

Source: Own material.

Reference should be made to the average vehicle occupancy per line route, when we analyse the average bus occupancy at individual bus stops of each line (Table 1). It is obvious that the average capacity of buses in Tab. 1 and 2 are identical, also the average bus occupancy for individual lines in these tables (MBP and MBC columns, respectively) show the differences that can be considered insignificant (in 6 cases it exceeds $1 \mathrm{pp}$, including 4 cases $2 \mathrm{pp}$, the biggest difference for the line $25 \mathrm{a}$ is $3.3 \mathrm{pp}$ ). This is due to the differences in the number of stops (route length) on individual line routes. Also the differences in the average number of passengers are not significant (columns MC and MP, the largest for lines 25a, 25b, 18, 14b -4.1, 1.8, 1.6 and 2.3, respectively). However, the differences are important in the case of position statistics and measures of dispersion.

Among the four lines in which the occupancy exceeds $50 \%$, three are the same lines as in the case of the occupancy on the routes - No. $7 \mathrm{~b}, 12 \mathrm{a}$ and $24 \mathrm{a}$, additionally on line $12 \mathrm{~b}$ the level of $50 \%$ has also been exceeded (in Table 1 this value is $49,6 \%$ ). For line No. 18, the occupancy is $49.4 \%$ (in Table $2-52 \%$ ). In the case of line No. 12 , the differences between lines $12 \mathrm{a}$ and $12 \mathrm{~b}$ are small, only the larger differences are: the minimum occupancy $-36.7 \%$ and $29.1 \%$, respectively; Q1-43.9\% and 39.4\% and the number of passengers - 80 and 74.8). Similarly, as before, the differences between lines $7 \mathrm{a}$ and $7 \mathrm{~b}$ and $24 \mathrm{a}$ and $24 \mathrm{~b}$ are very clear and these are basically the only lines for which significant differences are observed (only for lines 
No. 3 and 5 a difference of $6.5 \mathrm{pp}$ of the average occupancy and $9.6 \mathrm{pp}$ maximum occupancy on the buses can be considered as significant).

The largest average occupancy of the buses at individual stops of a given line was the highest on the route of line No. $7 \mathrm{~b}$ and amounted to $59 \%$. The minimum average occupancy at stops during the day on this line was $39.2 \%$, the median $60.8 \%$ and the maximum $68.5 \%$ (in Table 1 it was $23.2 \%$ and $86.8 \%$, respectively) and the coefficient of variation $14.4 \%$. The occupancy of the buses at stops of this line within a day differed on average by 8.5 percentage points from the average bus occupancy at individual stops within 24 hours and $75 \%$ of buses operated with the average occupancy of at least $56.9 \%$. Buses transported on average 82 people (in table 2 this value is 81.6 ).

A much lower level of the average occupancy at stops was on line No. $7 \mathrm{a}$. The average daily occupancy at bus stops was $45 \%$, and the average number of people traveling on the bus at individual stops was 67 . The maximum average occupancy per day was $60.1 \%$, and the minimum $27.2 \%$.

The second largest level of the average bus occupancy at individual stops was recorded on line No. $24 \mathrm{a}$ equal to $54.9 \%$, with the minimum average bus occupancy equal to $39 \%$ and the maximum of $63.2 \%$, and the coefficient of variation $11.1 \%$. At least half of the buses at individual stops of line $24 \mathrm{a}$ were filled in $57 \%$ and $75 \%$ at least in $52.9 \%$. The average daily number of passengers on the bus at the stop of this line was 62 people with the average nominal vehicle capacity of 115 people. It is significant that for line $24 \mathrm{~b}$ (the opposite direction to $24 \mathrm{a}$ ) the average daily occupancy at stops was lower by as much as $17.9 \mathrm{pp}(34.2 \%)$ and the number of passengers by nearly 23 people (39.3).

The smallest average level of bus occupancy at individual stops was recorded on the routes of lines $19 b-19.8 \%$ and $19 a-21.6 \%$. Comparing the data from Tab. 2 and Tab. 1 it is noted that a significant difference in the value of the parameters occurs for the coefficient of variation for both the occupancy and the number of passengers - for bus occupancy at stops it is twice less than for bus occupancy for routes. The same situation occurs for lines No. 2a and $2 \mathrm{~b}$ (only the average bus occupancy for these lines is less than $30 \%$ ).

Figure 4 presents the data on the average bus occupancy at individual stops of the No. 7 line. The chart clearly shows a smaller variation in the case of line No. $7 \mathrm{~b}$, which is practically on a constant level and amounts to $60 \%$, which increases slightly for stops in the city centre (including the railway station) and visibly falls for the three final stops at the Michałów estate (one of the largest residential areas of Radom and having a significant number of alternative connections, including those running along the same route - from the Chrobrego / Mierzejewskiego stop). In the case of line $7 \mathrm{a}$, the variation is very clear and the occupancy increases significantly in the middle part of the line running through the broad centre of Radom. It is probably important to note that the Chrobrego/Czysta stop is located at the largest shopping centre in Radom, and the Wierzbicka/Czarnoleska stop is a "node" stop located by the branching of the access roads to two parts of the South estate (significantly apart from each other, more than a dozen thousand residents live in each of them). 
Figure 4. Average occupancy of buses at individual bus stops: $a-$ No. 7a, b- No. 7b
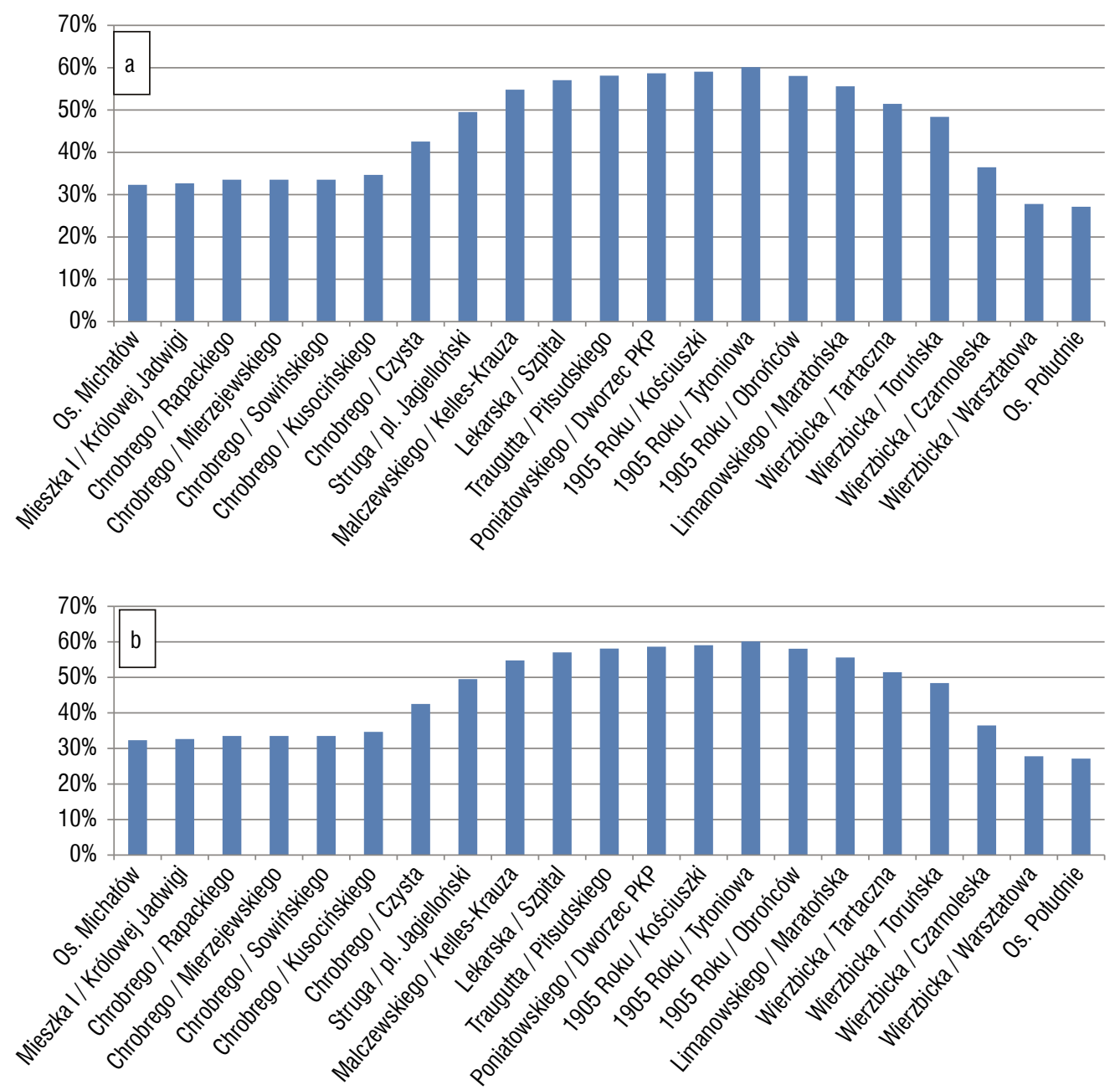

Source: Own material.

The average occupancy of public transport buses at individual stops of lines No. 11a and $11 \mathrm{~b}$ is shown in Figure 5. As in the case of Fig. 3, it can be assumed that the distribution of occupancy is identical. 
Figure 5. Average occupancy of buses at individual bus stops: $a-$ No. 11a, b- No. 11b
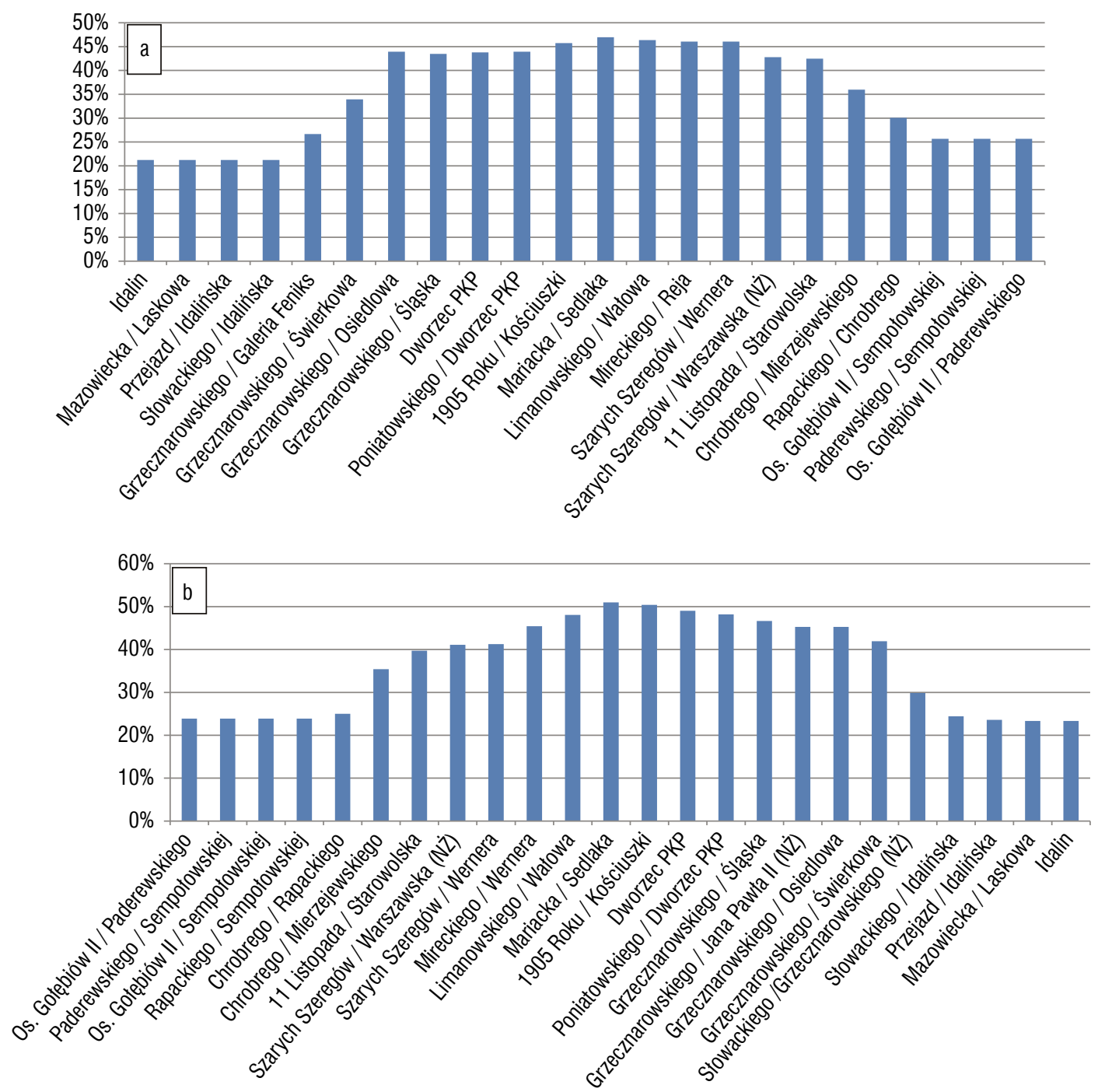

Source: Own material.

\section{The use of means of public transport by the inhabitants of Radom based on the surveys in households}

A survey was conducted among households in Radom as part of the project [Ciszewski T., et al., 2014], which was aimed at obtaining information on the mobility of the inhabitants of Radom and the surrounding area. The respondents indicated all journeys made on the working day preceding the day of the survey, in the so-called "travel diary". The study involved people over 12 years of age. The surveys were conducted in 1747 households in Radom (out of 70615 existing), receiving answers from 2913 respondents (1635 women and 1278 men). Drawing 
of proportional layered households (the layer was made up of households with 1, 2, 3, 4, 5+ household members). There were 186,987 people over the age of $13(99,822$ women, 87,005 men) in Radom (according to the database of the Universal Electronic System of Population Register (PESEL)). The surveyed households were inhabited by a total of 5,187 people, of which $46.53 \%$ were men, and $53.47 \%$ were women.

A resident of Radom most often traveled by car as a driver, on foot and by public transport. Estimated on the basis of the surveys, the average mobility of Radom's residents over 13 years old was at the level of 2.782 trips made by one person during the day and the total number of journeys amounted to 520.2 thousand. These values in the case of public transport are respectively 0.659 and 123.3 thousand (Table 3 ).

About 25\% of Radom's residents use public transport (Table 3), with very big differences due to the gender and age of the inhabitants. A definitely higher percentage of women use public transport than men (a difference of over $14 \mathrm{pp}$ ) in each age category. A particularly large difference occurs in the category of 45-

59 years (nearly $25 \mathrm{pp}$ ) and 13-17 years (nearly $21 \mathrm{pp}$ ). While it could have been expected that there would be a high rate of travel made by public transport among young people, the fact that only $13 \%$ of men aged $45-59$ use public transport is a surprise. The consequence of this is that the average daily number of trips by public transport carried out by a female resident of Radom is 0.812 and by a male inhabitant is 0.483 ; women make 81 thousand daily trips and men 42 thousand. ${ }^{4}$ The survey included complex journeys, i.e. carried out with at least two means of transport. ${ }^{5}$ In the case of public transport, the percentage of composite trips amounted to $34.9 \%$. Let us note that the highest was in the group of people aged 45-59, both among men and women. It draws attention to the fact that it was the lowest among women aged 13-17 years, at the same time in this group is the highest proportion using public transport.

Table 3. The percentage of the respondents [\%] traveling by public transport based on real journeys

\begin{tabular}{|c|c|c|c|c|c|c|c|c|c|c|c|c|c|c|c|}
\cline { 2 - 16 } \multicolumn{1}{c|}{ Women } & \multicolumn{4}{c|}{ Men } & \multicolumn{4}{c|}{ Women+Men } \\
\cline { 2 - 16 } & $13-17$ & $18-44$ & $45-59$ & $60+$ & Together & $13-17$ & $18-44$ & $45-59$ & $60+$ & Together & $13-17$ & $18-44$ & $45-59$ & $60+$ & Together \\
\hline 1 & 59.38 & 29.67 & 37.60 & 37.68 & 34.76 & 38.57 & 20.06 & 12.92 & 23.95 & 20.36 & 48.51 & 25.17 & 27.49 & 32.35 & 28.44 \\
\hline 2 & 1.234 & 0.660 & 0.865 & 0.906 & 0.812 & 0.771 & 0.496 & 0.324 & 0.540 & 0.483 & 0.997 & 0.577 & 0.615 & 0.763 & 0.659 \\
\hline 3 & 5969 & 26867 & 19972 & 28399 & 81207 & 3937 & 20888 & 6387 & 10837 & 42049 & 9907 & 47755 & 26358 & 39235 & 123256 \\
\hline 4 & 20.25 & 32.48 & 40.41 & 33.60 & 33.92 & 29.63 & 33.83 & 51.14 & 36.62 & 36.79 & 23.98 & 33.07 & 43.01 & 34.43 & 34.90 \\
\hline
\end{tabular}

\footnotetext{
1 - percentage of the respondents who travel by public transport based on real travel [\%];

2 - average daily number of journeys made by public transport of an inhabitant of Radom [number of trips/person];

3 - estimated daily number of journeys made by public transport;

4 - percentage of journeys made by public transport as part of a combined journey (at least two means of transport).

Source: Own material.
}

4 It is almost twice as much, although the ratio of the average daily number of trips made respectively by women and men is 1.68 - this is due to the fact that there are nearly 13,000. more women than men.

5 The walk was treated as a 'transport'. The trip is one journey in the statistics, although when determining the number of journeys, the given means of transport was included in the journey for each of the used means of transport. 
Travel motivations of the residents of Radom in the questionnaire studies were analysed. Eight source/destination motivations were distinguished:

1. House: place of residence;

2. Work: place of starting work;

3. Education: schools, colleges, place of courses, training;

4. Shopping and services: to a kiosk, shop, shopping centre;

5. Recreation and entertainment: for doing sports, to the cinema and restaurants;

6. Business matters: all trips made as part of work;

7. Offices, hospitals, clinics, banks, courts: performed not as part of work;

8. Other purposes: e.g. go to visit/to collect somebody, drive someone.

The tables present the average daily number of journeys by public transport according to the source of journey (Table 4) and destination (Table 5).

Table 4. Average daily number of journeys of the inhabitants of Radom and the share of [\%] journeys according to the beginning of the journey made by public transport based on real journeys

\begin{tabular}{|c|c|c|c|c|c|c|c|c|c|c|c|c|c|c|c|c|}
\hline B & A & $\begin{array}{c}\text { Share } \\
{[\%]}\end{array}$ & V & $\begin{array}{c}\text { Share } \\
{[\%]}\end{array}$ & V & $\begin{array}{c}\text { Share } \\
{[\%]}\end{array}$ & V & $\begin{array}{c}\text { Share } \\
{[\%]}\end{array}$ & A & \begin{tabular}{|c|} 
Share \\
{$[\%]$}
\end{tabular} & V & \begin{tabular}{|c|} 
Share \\
{$[\%]$}
\end{tabular} & V & $\begin{array}{c}\text { Share } \\
{[\%]}\end{array}$ & V & $\begin{array}{c}\text { Share } \\
{[\%]}\end{array}$ \\
\hline & \multicolumn{2}{|c|}{$\begin{array}{c}\text { Women } \\
13-17 \text { years }\end{array}$} & \multicolumn{2}{|c|}{$\begin{array}{c}\text { Women } \\
\text { 18-44 years }\end{array}$} & \multicolumn{2}{|c|}{$\begin{array}{c}\text { Women } \\
45-59 \text { years }\end{array}$} & \multicolumn{2}{|c|}{$\begin{array}{c}\text { Women } \\
60+\text { years }\end{array}$} & \multicolumn{2}{|c|}{$\begin{array}{c}\text { Men } \\
13-17 \text { years }\end{array}$} & \multicolumn{2}{|c|}{$\begin{array}{c}\text { Men } \\
\text { 18-44 years }\end{array}$} & \multicolumn{2}{|c|}{$\begin{array}{c}\text { Men } \\
\text { 45-59 years }\end{array}$} & \multicolumn{2}{|c|}{$\begin{array}{c}\text { Men } \\
60+\text { years }\end{array}$} \\
\hline 1 & 0.625 & 50.63 & 0.312 & 47.33 & 0.401 & 46.31 & .428 & 47.20 & 0.386 & 50.00 & 0.229 & 46.11 & 0.151 & 46.59 & 0.270 & 50.00 \\
\hline 2 & 200 & 0.00 & 12 & 17.03 & 81 & 20.94 & 7 & 1.87 & 10 & 0.00 & 53 & 0.78 & 077 & 3.86 & 030 & 5.63 \\
\hline 3 & 250 & 20.25 & 61 & 9.31 & 003 & 0.29 & & 0.00 & 229 & 29.63 & .067 & 13.47 & .000 & 0.00 & .000 & 0.00 \\
\hline 4 & 141 & 11.39 & 0.068 & 10.30 & 0.115 & \begin{tabular}{|l}
13.27 \\
\end{tabular} & 0.152 & 16.80 & 043 & 5.56 & 0.030 & 5.99 & 0.015 & 4.55 & 0.061 & 11.27 \\
\hline 5 & 109 & 8.86 & 038 & 5.74 & 026 & 2.95 & 027 & 2.93 & 100 & 12.96 & 0.046 & 9.28 & 0.011 & 3.41 & .019 & 3.52 \\
\hline 6 & 0.000 & 00 & 0.014 & 2.18 & 0.015 & 1 & 0.000 & 0 & 0 & 0 & 3 & 0.60 & 0 & 0 & 4 & 0.70 \\
\hline$T$ & 016 & 1.27 & 021 & 3.17 & 0.064 & 7.37 & 138 & 15.20 & .000 & 0.00 & 0.012 & 2.40 & 0.040 & 12.50 & 0.080 & 14.79 \\
\hline \multirow[t]{3}{*}{8} & 094 & 7.59 & 033 & 4.95 & 061 & 7.08 & 145 & 16.00 & 014 & 1.85 & 0.056 & 11.38 & 0.029 & 9.09 & .076 & 14.08 \\
\hline & 234 & 100 & 60 & 100 & 365 & 100 & 306 & 100 & 771 & 100 & 496 & 100 & 324 & 100 & 540 & 100 \\
\hline & \multicolumn{2}{|c|}{$\begin{array}{c}W+M \\
13-17 \text { years }\end{array}$} & \multicolumn{2}{|c|}{$\begin{array}{c}W+M \\
18-44 \text { years }\end{array}$} & \multicolumn{2}{|c|}{$\begin{array}{c}W+M \\
45-59 \text { years }\end{array}$} & \multicolumn{2}{|c|}{$\begin{array}{c}W+M \\
60+\text { years }\end{array}$} & \multicolumn{2}{|c|}{$\begin{array}{l}\text { W13+ } \\
\text { years }\end{array}$} & \multicolumn{2}{|c|}{$\begin{array}{c}M 13+ \\
\text { years }\end{array}$} & \multicolumn{2}{|c|}{$\begin{array}{c}\mathrm{W}+\mathrm{M} 13+ \\
\text { years }\end{array}$} & & \\
\hline 1 & 502 & 50.38 & 70 & 46.79 & 85 & 46.38 & 6 & 47.97 & 34 & 47.28 & 30 & 46.11 & 0.312 & 17 & & \\
\hline 2 & 000 & 0.00 & 082 & 14.30 & 133 & 21.65 & 022 & 2.91 & .093 & 11.44 & .050 & 10.78 & 0.073 & 11.09 & & \\
\hline 3 & 0.239 & 23.98 & 64 & 11.13 & 01 & 0.22 & 0 & 0.00 & 38 & 4.64 & 0.046 & 13.47 & 041 & 6.29 & & \\
\hline 4 & 0.090 & 9.07 & 0.049 & 8.41 & 0.069 & 11.16 & 0.117 & 15.27 & 0.109 & 13.38 & 0.034 & 5.99 & 0.074 & 11.24 & & \\
\hline 0 & 105 & 49 & 42 & 7.29 & 0 & 3.0 & 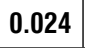 & 3.10 & 5 & 4.30 & 5 & 9.28 & 5 & 5. & & \\
\hline 6 & 0.000 & 0.00 & 9 & 1.49 & 8 & 1.34 & 001 & 0.19 & 009 & 1.16 & 002 & 0.60 & 006 & 0.92 & & \\
\hline 7 & 0.008 & 0.76 & 016 & 2.83 & 0.053 & 8.62 & 0.115 & 15.09 & 0.067 & 8.27 & 0.033 & 2.40 & 0.051 & 7.80 & & \\
\hline 8 & 0.053 & 5.31 & 045 & 7.76 & 0.047 & 7.57 & 18 & 15.47 & 077 & 9.53 & 0.052 & 11.38 & 066 & 9.98 & & \\
\hline & 0.997 & 100 & 0.577 & 100 & 0.615 & 100 & 0.763 & 100 & 0.794 & 100 & 0.483 & 100 & 0.659 & 100 & & \\
\hline
\end{tabular}

$\mathrm{B}$ - the beginning of the journey, A - average number of journeys

Source: Own material. 
A vast majority of journeys made by the residents of Radom began at their place of residence (1) $-47.37 \%$, and over $11 \%$ from the workplace (insignificant differences between the sexes and age groups). The significant differences between the sexes occur in the case of returning from shopping (4) and offices and hospitals (7) - the prevalence of women, while the large preponderance of men - from school (3) and recreation and entertainment (5). Taking into account the age groups and gender, we notice significant differences - some of them are natural for example: school-age people travel to school and do not go to work and do not travel for business purposes, on the contrary to the people of working age. It is also natural to observe a large share of people over 60 in returning from hospitals and outpatient clinics (7) and negligible business matters (6), but it is striking to have a large share in position (8) - other purposes. On the basis of the research, it is also possible to analyse the destinations of Radom's inhabitants in various age groups (Table 5).

Table 5. Average daily number of journeys of the inhabitants of Radom and share of [\%] journeys according to the destination of their journey made by public transport based on real journeys

\begin{tabular}{|c|c|c|c|c|c|c|c|c|c|c|c|c|c|c|c|c|}
\hline D & A & $\begin{array}{c}\text { Share } \\
{[\%]}\end{array}$ & V & $\begin{array}{c}\text { Share } \\
{[\%]}\end{array}$ & V & $\begin{array}{c}\text { Share } \\
{[\%]}\end{array}$ & V & $\begin{array}{c}\text { Share } \\
{[\%]}\end{array}$ & A & $\begin{array}{c}\text { Share } \\
{[\%]}\end{array}$ & V & $\begin{array}{c}\text { Share } \\
{[\%]}\end{array}$ & V & $\begin{array}{c}\text { Share } \\
{[\%]}\end{array}$ & V & $\begin{array}{c}\text { Share } \\
{[\%]}\end{array}$ \\
\hline & \multicolumn{2}{|c|}{$\begin{array}{c}\text { Women } \\
13-17 \text { years }\end{array}$} & \multicolumn{2}{|c|}{$\begin{array}{c}\text { Women } \\
\text { 18-44 years }\end{array}$} & \multicolumn{2}{|c|}{$\begin{array}{c}\text { Women } \\
45-59 \text { years }\end{array}$} & \multicolumn{2}{|c|}{$\begin{array}{c}\text { Women } \\
60+\text { years }\end{array}$} & \multicolumn{2}{|c|}{$\begin{array}{c}\text { Men } \\
\text { 13-17 years }\end{array}$} & \multicolumn{2}{|c|}{$\begin{array}{c}\text { Men } \\
\text { 18-44 years }\end{array}$} & \multicolumn{2}{|c|}{$\begin{array}{c}\text { Men } \\
\text { 45-59 years }\end{array}$} & \multicolumn{2}{|c|}{$\begin{array}{c}\text { Men } \\
60+\text { years }\end{array}$} \\
\hline 1 & 0.594 & 48.10 & 0.288 & 43.56 & 395 & 45.72 & 418 & 46.13 & 386 & 50.00 & 0.229 & 46.11 & 0.154 & 47.73 & 0.236 & 43.66 \\
\hline 2 & 00 & 0.00 & 35 & 0.40 & 189 & 1.83 & 022 & 2.40 & 000 & 0.00 & 056 & 11.38 & .063 & 19.32 & .038 & 7.0 \\
\hline 3 & 297 & 4.05 & 0.061 & 9.31 & 0.005 & 0.59 & 000 & 0.00 & 0.243 & 31.48 & 0.070 & 14.07 & 0.000 & 0.00 & 0.000 & 0.0 \\
\hline 4 & 156 & 2.66 & 667 & 0.10 & 105 & 12.09 & 157 & 17.33 & 029 & 3.70 & 0.028 & 5.69 & 011 & 3.41 & 0.068 & 12.6 \\
\hline 5 & 99 & 7.59 & 039 & 5.94 & 036 & 4.13 & 029 & 3.20 & 0.100 & 12.96 & 0.052 & 10.48 & 0.015 & 4.55 & 0.019 & 3.5 \\
\hline 6 & 00 & 0.00 & 0.008 & 1.19 & 0.013 & 1.47 & .000 & 0.00 & 0.000 & 0.00 & 0.000 & 0.00 & 0.011 & 3.41 & 0.004 & 0.7 \\
\hline 7 & 0.016 & 1.27 & 6 & 3.96 & 66 & 7.67 & 35 & 14.93 & 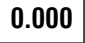 & 0.00 & 0.012 & 2.40 & 37 & 11.36 & 91 & 16. \\
\hline \multirow[t]{3}{*}{8} & 78 & 6.33 & 0.037 & 5.54 & 056 & 6.49 & 145 & 16.00 & 0.014 & 1.85 & 0.049 & 9.88 & 0.033 & 10.23 & 0.084 & 15.4 \\
\hline & 234 & 100 & 660 & 100 & 0.865 & 100 & .906 & 100 & 771 & 100 & 0.496 & 100 & 0.324 & 100 & 0.540 & 100 \\
\hline & \multicolumn{2}{|c|}{$\begin{array}{c}W+M \\
13-17 \text { years }\end{array}$} & \multicolumn{2}{|c|}{$\begin{array}{c}W+M \\
18-44 \text { years }\end{array}$} & \multicolumn{2}{|c|}{$\begin{array}{c}W+M \\
45-59 \text { years }\end{array}$} & \multicolumn{2}{|c|}{$\begin{array}{c}W+M \\
60+\text { years }\end{array}$} & \multicolumn{2}{|c|}{$\begin{array}{l}\text { W13+ } \\
\text { years }\end{array}$} & \multicolumn{2}{|c|}{$\begin{array}{c}\text { M 13+ } \\
\text { years }\end{array}$} & \multicolumn{2}{|c|}{$\begin{array}{c}\mathrm{W}+\mathrm{M} 13+ \\
\text { years }\end{array}$} & & \\
\hline 1 & 00 & 48.86 & 258 & .68 & 284 & 46.21 & 347 & 45.45 & 0.368 & 45.33 & 0.223 & 46.09 & 0.300 & 45.59 & & \\
\hline 2 & 00 & 0.00 & 95 & 6.45 & 131 & 21.22 & 028 & 3.68 & 105 & 12.96 & 0.050 & 10.40 & 0.080 & 12.08 & & \\
\hline 3 & 69 & 27.00 & 66 & .39 & 003 & 0.45 & 000 & 0.00 & 0041 & 4.99 & 0.048 & 9.94 & 0.044 & 6.68 & & \\
\hline 4 & 91 & 9.10 & 47 & 17 & 1 & 9.99 & 22 & 16.05 & 88 & 13.31 & 0 & 6.96 & 73 & 11.14 & & \\
\hline 5 & 0.097 & 9.73 & 0.046 & 7.93 & 0.026 & 4.23 & 0.025 & 3.29 & 0.038 & 4.66 & 0.039 & 8.02 & 0.038 & 5.80 & & \\
\hline 0 & 0.000 & 0.00 & 0.004 & 0.67 & 0.012 & 1.94 & 1 & 0.19 & 0.000 & 0.76 & 0.000 & 0.70 & 0.005 & 0.74 & & \\
\hline 7 & 0.008 & 0.76 & 0.019 & 8 & 3 & 8.56 & 8 & 15.48 & 9 & 8.51 & 0 & 7.27 & 0.053 & 8.09 & & \\
\hline 8 & 0.045 & 4.55 & 0.043 & 7.44 & .046 & 7.40 & 0.121 & 15.86 & 0.077 & 9.49 & 0.051 & 10.63 & 0.065 & 9.88 & & \\
\hline & 0.997 & 100 & 0.577 & 100 & 0.615 & 100 & 763 & 100 & 0.812 & 100 & 0.483 & 100 & 0.659 & 100 & & \\
\hline
\end{tabular}

$\mathrm{D}$ - destination, A - average number of journeys

Source: Own material. 
The day before the survey, the main destination of the journey by public transport of the inhabitants of Radom was their house $-45.59 \%$, then it was work $12.80 \%$. Note that these values differ by about $2 \mathrm{pp}$ from the values in Table 4 - an important destination of travel from work is a different goal than home, also part of the journey to work does not take place from home.

Another motivation for the inhabitants of Radom to travel by bus was shopping and various services, accounting for $11.14 \%$ of all the trips. Another destination: go to visit/to collect somebody or drive someone (8) accounted for $9.88 \%$ of all the trips, while journeys to offices and hospitals accounted for $8.09 \%$ of all the journeys recorded.

Table 6 contains selected destinations and their share in the number of trips by public transport carried out by women. In the group of women aged 18-44, only for the homework trips, the average daily number of trips exceeds 0.1 and the same situation occurs if we do not include the age. In the 13-17 age group, we have five such destinations (in total over $76 \%$ of the journeys), for $45-59$ years of age - 3 destinations $(50.1 \%)$ and for over 60 years of age - six destinations (83.5\%). Theoretically there were 64 kinds of trips. In the case of men, only in the 13-17 age group there was a situation where the average daily number of trips by public transport was not less than 0.1 trips. These were home-education, education-home and entertainment-home destinations, accounting for $74.1 \%$ of all the journeys. Taking into account the age groups but not distinguishing between sexes in the group of 13-18 years of age, three destinations (house-education, education-house, shopping-house, $61.5 \%$ ) meet the condition "the average daily number of trips by public transport was not less than 0.1 trip", in the 45-59 age group - two destinations (house-work, work-home, 38.4\%), in the age group over 60 years of age - four destinations $(56.2 \%)$.

Table 6. Average daily number of journeys by public transport and their share [\%] in direct trips made by women in individual age groups based on real journeys*

\begin{tabular}{|c|c|c|c|c|c|c|c|c|c|c|c|}
\hline & \multirow[t]{2}{*}{ Trips } & \multicolumn{2}{|c|}{$13-17$ years } & \multicolumn{2}{|c|}{ 18-44 years } & \multicolumn{2}{|c|}{$45-59$ years } & \multicolumn{2}{|c|}{$60+$ years } & \multicolumn{2}{|c|}{$13+$ years } \\
\hline & & $x$ & $y$ & $x$ & $y$ & $x$ & $y$ & $x$ & $y$ & $x$ & y \\
\hline $1-3$ & Home - Education & 0.297 & $24.1 \%$ & 0.059 & $8.9 \%$ & 0.005 & $0.6 \%$ & 0.000 & $0.0 \%$ & 0.040 & $5.1 \%$ \\
\hline $1-4$ & Home - Shopping & 0.141 & $11.4 \%$ & 0.039 & $5.9 \%$ & 0.071 & $8.3 \%$ & 0.126 & $13.9 \%$ & 0.073 & $9.2 \%$ \\
\hline $3-1$ & Education - Home & 0.250 & $20.3 \%$ & 0.047 & $7.1 \%$ & 0.003 & $0.3 \%$ & 0.000 & $0.0 \%$ & 0.032 & $4.1 \%$ \\
\hline $4-1$ & Shopping - & 0.141 & $11.4 \%$ & 0.060 & $9.1 \%$ & 0.105 & $\%$ & 0.138 & $15.2 \%$ & 0.094 & $1.8 \%$ \\
\hline $5-1$ & Recreation - Home & 0.109 & $8.9 \%$ & 0.037 & $5.5 \%$ & 0.020 & $2.4 \%$ & 0.027 & $2.9 \%$ & 0.033 & $4.2 \%$ \\
\hline $1-2$ & Home - Work & 0.000 & $0.0 \%$ & 0.124 & $18.8 \%$ & 0.179 & $20.6 \%$ & 0.022 & $2.4 \%$ & 0.106 & $13.4 \%$ \\
\hline $2-1$ & Work - Home & 0.000 & $0.0 \%$ & 0.094 & $14.3 \%$ & 0.151 & $17.4 \%$ & 0.017 & $1.9 \%$ & 0.084 & $10.6 \%$ \\
\hline $1-7$ & Home - Offices/ hospitals & 0.016 & $1.3 \%$ & 0.021 & $3.2 \%$ & 0.056 & $6.5 \%$ & 0.118 & $13.1 \%$ & 0.054 & $6.8 \%$ \\
\hline $1-8$ & Home - Other purposes & 0.078 & $6.3 \%$ & 0.031 & $4.8 \%$ & 0.048 & $5.6 \%$ & 0.138 & $15.2 \%$ & 0.064 & $8.1 \%$ \\
\hline $7-1$ & Offices/hospitals - Home & 0.016 & $1.3 \%$ & 0.016 & $2.4 \%$ & 0.056 & $6.5 \%$ & 0.109 & $12.0 \%$ & 0.049 & $6.2 \%$ \\
\hline $8-1$ & Other purposes - Home & 0.078 & $6.3 \%$ & 0.026 & $4.0 \%$ & 0.048 & $5.6 \%$ & 0.128 & $14.1 \%$ & 0.059 & $7.5 \%$ \\
\hline
\end{tabular}

$\mathrm{x}$ - average daily number of journeys, $\mathrm{y}$ - travel share in the journeys in a given age group

* Consider only these trips in which even in one group the average daily number of trips is not less than 0.1 .

Source: Own material. 
Men not only used public transport less frequently, but also the number of motivations for traveling (source-destination) is much smaller than for women (Table 8). Please note that among 64 motivations $^{6}$, the respondents indicated only 45 motivations - women 43, men 34; so men pointed out two motivations that did not occur among women: recreation-work, shopping-recreation.

Table 7. Average daily number of journeys by public transport and the share [\%] in the direct trips made by a) men, b) men and women in individual age groups based on real journeys*

\begin{tabular}{|c|c|c|c|c|c|c|c|c|c|}
\hline \multirow[t]{2}{*}{ Trips } & \multicolumn{2}{|c|}{$\begin{array}{c}M \\
13-17 \text { years }\end{array}$} & \multirow[t]{2}{*}{ Trips } & \multicolumn{2}{|c|}{$\begin{array}{c}W+M \\
13-17 \text { years }\end{array}$} & \multicolumn{2}{|c|}{$\begin{array}{c}W+M \\
45-59 \text { years }\end{array}$} & \multicolumn{2}{|c|}{$\begin{array}{c}W+M \\
60+\text { years }\end{array}$} \\
\hline & $x$ & y & & $x$ & y & $x$ & y & $x$ & y \\
\hline Home - Education & 0.243 & $31.5 \%$ & Home - Education & 0.269 & $27.0 \%$ & 0.003 & $0.4 \%$ & 0.000 & $0.0 \%$ \\
\hline Education - Home & 0.229 & $29.6 \%$ & Education - Home & 0.239 & $24.0 \%$ & 0.001 & $0.2 \%$ & 0.000 & $0.0 \%$ \\
\hline Receation - Home & 0.100 & $13.0 \%$ & Shopping - Home & 0.105 & $10.5 \%$ & 0.061 & $10.5 \%$ & 0.105 & $13.7 \%$ \\
\hline & & & Home - Work & 0.000 & $0.0 \%$ & 0.125 & $20.3 \%$ & 0.025 & $3.3 \%$ \\
\hline & & & Work - Home & 0.000 & $0.0 \%$ & 0.112 & $18.1 \%$ & 0.021 & $2.7 \%$ \\
\hline & & & Home - Other purposes & 0.008 & $0.8 \%$ & 0.038 & $6.2 \%$ & 0.106 & $13.9 \%$ \\
\hline & & & Offices/Hospital - Home & 0.008 & $0.8 \%$ & 0.049 & $7.9 \%$ & 0.114 & $14.9 \%$ \\
\hline & & & Other purposes - Home & 0.045 & $4.5 \%$ & 0.038 & $6.2 \%$ & 0.105 & $13.7 \%$ \\
\hline
\end{tabular}

$\mathrm{x}$ - average daily number of journeys, $\mathrm{y}$ - travel share in the journeys in a given age group

* Consider only these trips in which, in one group (separately for men and separately for men and women), the average daily number of trips is not less than 0.1 . In the non-existent groups there were no trips that met this condition.

Source: Own material.

Table 8. Number of source-destinations in journeys by means of public transport in particular age groups, including gender

\begin{tabular}{|c|c|c|c|c|c|c|c|c|c|c|c|c|c|c|}
\hline \multicolumn{4}{|c|}{ Women } & \multicolumn{4}{c|}{ Men } & \multicolumn{5}{c|}{ Women + Men } \\
\hline $13-17$ & $18-44$ & $45-59$ & $60+$ & Together & $13-17$ & $18-44$ & $45-59$ & $60+$ & Together & $13-17$ & $18-44$ & $45-59$ & $60+$ & Together \\
\hline 11 & 36 & 31 & 19 & 43 & 8 & 29 & 16 & 17 & 34 & 11 & 42 & 31 & 23 & 45 \\
\hline
\end{tabular}

Source: Own material.

\section{Summary}

An efficient transport system that requires rational separation of transport tasks is important from the point of view of efficient functioning of urban agglomerations. The most difficult task for carriers is to change transport preferences of residents and increase the share of public transport journeys in cities. The share of trips with the use of public transport accounts for only $23.7 \%$ of all the journeys in Radom (even less, because only $18.2 \%-94.6$ thousand if we

\footnotetext{
6 In this type $(\mathrm{x})-(\mathrm{x})$; there was no home-house or work-work trip among this type of trips.
} 
do not include combined journeys, when the bus was not the primary means of transport). This percentage is very low compared to Warsaw. Only $28.4 \%$ of the travelers (real journeys) used public transport in any form. At the same time, as much as $29 \%$ of Radom's residents (36.8\% men and $22.2 \%$ women) declare not using public transport at all (even sporadically). Considering these aspects, a relatively high general assessment of public transport is puzzling (in the same studies), which on a scale of 1 to 10 is 6.24 points. However, in terms of comfort, security, adjustment to the needs of disabled people and the functionality of transfer nodes ${ }^{7}$ is at the level of 5.5 points. One justification is the fact that more than $84 \%$ of the travelers, when choosing a means of transport, are guided primarily by the duration of their journey. Public transport in Radom is based exclusively on bus transport organized by MZDiK. There is no city rail transport or private transport with minibuses in Radom. The concepts of tramway transport (promoted by Professor Michał Kelles-Krauza or now by the Radom "Bicycle Brotherhood") have no chance of being realized, not only for financial reasons. Based on the same surveys, it was estimated that only $47.4 \%$ of the residents believe that tram lines would improve the quality of public transport, but at the same time more than $70 \%$ believe that high-priority routes for buses would improve such quality. However, it is problematic to assess what the respondents understood through high priority routes in the latter case.

The assessment of the effectiveness of the method used to measure the occupancy of public transport vehicles is difficult due to the lack of other (parallel) tests enabling the comparison of the results. An unquestionable advantage is the low cost of testing and the possibility of continuous measurement - completion of the questionnaire could be included in the scope of the responsibilities of drivers and the process of entering data should be automated. The disadvantage, as in many other methods, is the human factor - the subjectivity of the assessment, the significance of which will be lower and lower with the acquisition of experience by drivers. Difficult to assess is the fact that the fear of drivers that too low occupancy will result in the liquidation of lines and the loss of jobs (and such fears were put forward by the drivers in the interviews with the interviewers) affect the falsification of the assessment. This method is not effective for the assessment of the exchange of the number of passengers at bus stops. Although it is important to know that at certain stops and routes there is a total exchange of passengers and information on situations in which passengers who have not found a place on the bus remain at bus stops.

Bus lines are relatively long - a vast majority have more than 20 stops in Radom (Table 2). The vehicles' occupation and the number of passengers at the first stops are low and significantly increase in the middle parts of the routes - within the city centre. At the same time there are so-called transport bundles (doubling of the lines) and almost no interchanges. Perhaps the solution that would increase the efficiency and attractiveness of public transport would be the creation of interchange nodes on the outskirts of the very centre and the service of peripheral stops with buses of small capacity, and in the centre - with high capacity buses. However,

\footnotetext{
7 Accessibility assessment at the level of 6.4 points it is in some contradiction with this.
} 
this would have to do with the provision of time tickets with the time not less than the travel time of the longest (in terms of travel time) lines and the price not higher than a single-ticket.

\section{References}

1. Ciszewski T., Dębowska-Mróz M., Ferensztajn-Galardos E., Grad B., Krajewska R., Łukasik Z., Rogowski A., Wojciechowski W., 2014. Analiza przemieszczeń i preferencji komunikacyjnych na obszarze ROF opracowanie zespołu UTH Radom (umowa NR 1/KM4/2014 z dnia 21 maja 2014 r. między SITK Oddział w Krakowie a UTH w Radomiu) w ramach projektu Zintegrowane planowanie transportu zrównoważonego miejskiego Radomskiego Obszaru Funkcjonalnego (umowa MPU-II/3302/4/2014 z dnia 22.04.2014 r. między Miejską Pracownią Urbanistyczną w Radomiu a SITK Oddział w Krakowie).

2. Rudnicki A., 1991. Jakość komunikacji miejskiej. Kraków: SITK.

3. Rydzkowski W., Wojewódzka-Król K. (Eds.), 2000. Transport, Warszawa: PWN.

4. Starowicz W., 2007. Jakość przewozów w miejskim transporcie zbiorowym, Kraków: Politechnika Krakowska im. Tadeusza Kościuszki.

5. Tracz M. (Ed.), 1984. Pomiary i badania ruchu drogowego, Warszawa: WKiŁ.

6. Łukasik Z., Kuśmińska-Fijałkowska A, Żurek-Mordka M., 2016. Możliwości wykorzystania czujników ruchu w transporcie. Autobusy, No. 12, pp. 684-688.

7. Mitas W.A., Bernaś M., Bugdol M, Ryguła A., Konior W., 2011. Elektroniczne narzędzia pomiarowe w transporcie - wagi preselekcyjne. Elektronika, No. 12, pp. 86-89.

8. Ryguła A., Loga W., Brzozowski K., 2015. Estymacja napełnienia pojazdów komunikacji zbiorowej z wykorzystaniem preselekcyjnych systemów ważenia pojazdów. TTS, No. 12.

9. Morfoulaki, M., Tyrinopoulos, Y., Aifadopoulou, G., 2007. Estimation of satisfied customers in public transport systems: a new methodological approach. Journal of the Transportation Research Forum, Vol. 46, No. 1, pp. 63-72.

10. Friman, M., Fellesson, M., 2009. Service supply and customer satisfaction in public transportation: the quality paradox. Journal Public Transport. Vol. 12, No. 4, pp. 57-69.

11. Eboli, L., Mazzulla, G., 2009. A new customer satisfaction index for evaluating transit service quality. Journal Public Transport, Vol. 12, No. 3, pp. 21-38.

12. Guirao, B., García-Pastor, A., López-Lambas, M.E., 2016. The importance of service quality attributes in public transportation: narrowing the gap between scientific research and practitioners' needs. Transport Policy Vol. 49, pp. 68-77.

13. Wen-Tai Laia, Ching-Fu Chenb, 2011. Behavioral intentions of public transit passengersThe roles of service quality, perceived value, satisfaction and involvement. Transport Policy. Vol. 18, Iss. 2, pp. 318-325.

14. Banister D., 2008. The sustainable mobility paradigm. Transport Policy Vol. 15, pp. 73-80.

15. Givoni M., Banister D., 2010. Integrated transport, from policy to practice. London and New York: Rutledge Taylor\&Francis Group, pp. 5-11.

16. Hine J., 2000. Integration integration integration ... Planning for sustainable and integrated transport systems in the new millennium, Pergamon. Transport Policy, Vol. 7. 
17. Hull A., 2005. Integrated transport planning in the UK: from concept to reality. Journal of Transport Geography, No. 13, pp. 318-328.

18. Ibrahim M.F., 2003. Improvements and integration of a public transport system: the case of Singapore. Cities, Vol. 20, No. 3, pp. 205-216.

19. Preston J., 2010. What's so funny about peace, love and transport integration? Research in Transportation Economics, No. 29, pp. 329-338.

20. http://strategiatransportowa.um.warszawa.pl/sites/default/files/buspas_tl-opis.pdf [accessed: 1.3.2018]

21. http://www.infotron.com.pl/index.php/system-zliczania-pasazerow [accessed: 1.03.2018]

22. http://www.mzdik.radom.pl [accessed: 1.3.2018]

23. http://www.pixel.pl/systemy/system-automatycznego-zliczania-pasazerow/ [accessed: 1.3.2018]

24. www.letronik.cc.pl/liczniki/o771/o771InstrukcjaMontazu.pdf [accessed: 1.8.2018] 